\title{
Evidence for degraded low frequency verbal concepts in left resected temporal lobe epilepsy patients
}

\author{
${ }^{1}$ Visser, M., ${ }^{1}$ Forn, C., ${ }^{2}$ Lambon Ralph, M.A., ${ }^{3}$ Hoffman, P., ${ }^{4}$ Gómez Ibáñez, A., \\ ${ }^{5}$ Sanjuan-Tomas, A., ${ }^{1}$ Rosell Negre, P., ${ }^{4}$ Villanueva, V., ${ }^{1}$ Ávila, C.
}

${ }^{1}$ NNF, Departament de Psicología Bàsica, Clínica i Psicobiología, University Jaume I, Castellón, Spain.

${ }^{2}$ NARU, Division of Neuroscience and Experimental Psychology, University of Manchester, UK.

${ }^{3}$ School of Philosophy, Psychology and Language Sciences, University of Edinburgh, UK.

${ }^{4}$ Refractory Epilepsy Unit, Neurological Service, Hospital Universitario y Politécnico La Fe, Valencia, Spain

${ }^{5}$ Departament de Tecnologies de la Informació i les Comunicacions, University Pompeu Fabra, Barcelona, Spain

Figures: 7

Tables: 4

Pages: 36

Words Abstract: 210

Corresponding author: Maya Visser, department of PsicologíaBásica, Clínica y Psicobiología ,Universitat Jaume I, Avda. SosBaynat s/n, 12071, maya.visser@gmail.com, phone number: +349582246789, fax number: +34964729267

Acknowledgements: This research was supported by the Spanish Ministry of Science and Innovation (PSI2013-45378-R) and the University Jaume I, Castellon (P1·1B2014-03).

Conflicts of interest: none 


\begin{abstract}
According to a large neuropsychological and neuroimaging literature, the bilateral anterior temporal lobe (ATL) is a core region for semantic processing. It seems therefore surprising that semantic memory appears to be preserved in temporal lobe epilepsy (TLE) patients with unilateral ATL resection. However, recent work suggests that the bilateral semantic system is relatively robust against unilateral damage and semantic impairments under these circumstances only become apparent with low frequency specific concepts. In addition, neuroimaging studies have shown that the function of the left and right ATLs differ and therefore left or right ATL resection should lead to a different pattern of impairment. The current study investigated hemispheric differences in the bilateral semantic system by comparing left and right resected TLE patients during verbal semantic processing of low frequency concepts. Picture naming and semantic comprehension tasks with varying word frequencies were included to investigate the pattern of impairment. Left but not right TLE patients showed impaired semantic processing, which was particularly apparent on low frequency items. This indicates that, for verbal information, the bilateral semantic system is more sensitive to damage in the left compared to the right ATL, which is in line with theories that attribute a more prominent role to the left ATL due to connections with pre-semantic verbal regions.
\end{abstract}

Key words: epilepsy; language; semantic memory; temporal lobes 


\section{Introduction}

\subsection{Semantic memory}

Semantic memory refers to the meaning of words, pictures, sounds and general information about the world. Damage to the bilateral anterior temporal lobes (bATL) leads to impaired semantic performance in Alzheimer's disease, semantic dementia (SD) and herpes simplex encephalitis (HSE), suggesting that the bATL is a core region for semantic representation (Lambon Ralph, 2014; Lambon Ralph, Jefferies, Patterson, \& Rogers, 2017; Rogers et al., 2004). This is further supported by a range of functional neuroimaging studies in healthy participants (Devlin et al., 2000; Hoffman, Binney, \& Lambon Ralph, 2015; Humphreys et al., 2015; Vandenberghe, Price, Wise, Josephs, \& Frackowiak, 1996; Visser et al., 2010).

Classical models suggest that conceptual knowledge arises from networks of modality-specific brain regions distributed throughout the cortex (for reviews see: Capitani, Laiacona, Mahon, \& Caramazza, 2003; Humphreys \& Forde, 2001; Martin, 2007). The newer hub-and-spoke's model suggest that these modality specific (i.e., "spoke") regions interact with the transmodal ATL "hub", whose function is to distil coherent concepts from these "spoke" regions (Lambon Ralph, 2014; Lambon Ralph, Jefferies, Patterson, \& Rogers, 2017; Rogers et al., 2004). This model suggests that the brain must contain a central semantic hub to support generalizations across concepts that have similar conceptual relations but very different property profiles. For example, dogs and parrots look and behave very differently, yet share many conceptual relations that humans can easily use to support categorical generalizations (e.g., are animals, breath air, are pets etc.). The bATL is a good candidate to serve as a central hub as it connects with many regions throughout the brain (Binney, Parker, \& Lambon Ralph, 2012; Lambon Ralph et al., 2017; Rogers et al., 2004).

\subsection{Semantic impairments in left and right Temporal Lobe Epilepsy patients}

The ideas of Lambon Ralph, Patterson and colleagues originate from SD patient studies. The semantic impairments in SD patients are caused by progressive bilateral ATL atrophy. Therefore, studies on temporal lobe epilepsy (TLE) patients with unilateral left or right ATL lobectomy can advance our knowledge on the role of the bATL and specifically on differences between left and right ATL. However, studies on TLE 
patients without ATL lobectomy, in which dysfunction of this area is heterogeneous, show an inconsistent pattern on left/right differences. Whereas some studies have showed that left but not right TLE patients are impaired on expressive naming tasks (Drane et al., 2013; Giovagnoli, Erbetta, Villani, \& Avanzini, 2005; Voltzenlogel et al., 2006; Voltzenlogel, Hirsch, Vignal, Valton, \& Manning, 2015), other studies revealed naming impairments in both left and right TLE patients (Giovagnoli et al., 2016; Messas, Mansur, \& Castro, 2008; Seidenberg et al., 2002). Furthermore, the literature base on the semantic impairments in resected TLE patients is scarce. A few studies have examined remote memory problems using famous faces or scenes, thereby probing semantic memory (Barr et al., 1990; Drane et al., 2013; Lah et al., 2004).

Barr et al. (1990) were the first to describe that left but not right resected TLE patients were significantly impaired at recalling famous people's names. This result was replicated by Lah et al. in 2004, who found that naming deficits were evident in recall but not in recognition. Therefore, Lah et al.(2004) suggested that patients show a retrieval problem rather than damage to a semantic store. In addition, they showed that both left and right resected TLE patients were impaired when asked to answer questions about famous events. In the same study, these results were repeated on a verbal fluency task for famous persons and events: left TLE patients were impaired on the former, whereas both left and right TLE patients were impaired on the latter category. According to Lah et al. this fits with Damasio's model (1989), which suggests that the ATL is not involved in general semantics but is involved in knowledge for unique entities.

A recent study by Drane et al. (2013) provided another view. Their study showed a double dissociation: right resected TLE patients had problems recognizing faces but once recognized they could always name the famous person. In contrast, left TLE patients could often recognise a face but were unable to name it. Based on this, Drane et al., (2013) suggested that the right ATL plays a fundamental role in accessing semantic information from a visual route, whereas the left ATL serves to link semantic information to the language system, which is required for naming.

The studies above were limited to remote memory on faces and/or events. In contrast, two recent studies have showed semantic memory impairments in resected TLE patients using a new semantic battery which focused on probing low frequency concepts (Lambon Ralph et al., 2012; Rice, Caswell, Moore, Hoffman, \& Lambon 
Ralph, 2018). These studies showed that patients performed well on standard semantic tasks probing concepts at the basic level (although semantic impairment was reflected by a $\mathrm{n}$ increase in response times on these tasks). However, patients were impaired for more demanding concepts referring to specific and abstract information. Furthermore, in the former study both left and right TLE patients showed semantic impairments on verbal tasks, although these were more severe for left TLE patients (Lambon Ralph et al., 2012). However, in the latter study, semantic impairments were found in the left TLE but not in the right TLE patient group (Rice et al., 2018).

The authors of these two studies pointed out that many current standard semantic memory tests are not sensitive to mild semantic impairments in TLE as they tend to use highly frequent concepts. This is important as the observed absence of semantic problems in these patients has cast doubt on the semantic role of the ATL. Taking into account the well-defined episodic memory impairments in TLE patients, it is logical that these impairments dominate the research field taking away the focus from potential semantic problems. As such, some influential review articles have suggested that the semantic role of the ATL should be considered with caution as unilateral ATL lobectomy does not commonly lead to semantic impairments (Hickok \& Poeppel, 2004; Simmons \& Martin, 2009).

1.3 Models explaining the frequency and lateralization effects in the semantic system As explained above, the hub-and-spoke's model suggests that a transmodal hub in the bilateral ATL forms concepts through connections with modality-specific regions (Lambon Ralph et al., 2017; Rogers et al., 2004). The more frequently a concept is processed, the stronger its bilateral representation becomes, making it more readily available and more robust to damage. After unilateral damage, the remaining ATL can continue to support comprehension for strongly-instantiated high frequency concepts; thus small semantic impairments are easily overlooked on standard semantic tests (Bell \& Giovagnoli, 2007; Rice et al., 2018; Rice, Hoffman, \& Lambon Ralph, 2015; Rogers et al., 2004; Schapiro, McClelland, Welbourne, Rogers, \& Lambon Ralph, 2013). In other words, the remaining semantic system in the unilateral ATL is still able to construct highly frequent concepts but struggles when probing low frequent, specific concepts. 
In the first computational model of the hub-and-spoke's model, the left and right ATL represented conceptual knowledge in a unified manner as part of a bilateral coupled system (Rogers et al., 2004). In agreement, repetitive transcranial magnetic stimulation (rTMS) to either the left or right ATL results in equivalent levels of semantic impairment for both verbal and nonverbal semantic tasks (Lambon Ralph, Pobric, \& Jefferies, 2009; Pobric, Jefferies, \& Lambon Ralph, 2010; Pobric, Jefferies, \& Ralph, 2007). However, such a model is unable to explain the different neuropscyhological patterns seen in left and right TLE patients. Therefore, newer implementations of the model suggest that graded hemispheric specialisation emerges as a consequence of connectivity with modality-specific regions (Binney, Parker, \& Lambon Ralph, 2012; Rice, Caswell, Moore, Hoffman, \& Lambon Ralph, 2018; Rice, Hoffman, \& Lambon Ralph, 2015; Rice, Lambon Ralph, \& Hoffman, 2015; Schapiro, McClelland, Welbourne, Rogers, \& Lambon Ralph, 2013; Visser \& Lambon Ralph, 2011). For example, left $>$ right differences reflect the stronger connectivity of the ATL with the left-lateralized language network (for a more detailed explanation see the review of Rice et al., 2015). In line with this, a large meta-analysis of functional neuroimaging data showed that the bilateral ATL are involved in all tasks and modalities, with a relatively increased reliance on the left ATL during verbal production and comprehension (Rice, Hoffman, \& Lambon Ralph, 2015).

However, when considering verbal tasks, we need to distinguish between naming and comprehension tasks. Neuropsychological data from SD patients shows a left-side dominance during expressive tasks due to connections with left-hemisphere phonological regions, whereas this left lateralisation effect was less strong during comprehension tasks (Lambon Ralph, McClelland, Patterson, Galton, \& Hodges, 2001). This aligns with TLE studies that have shown that naming impairments are most prominent in left TLE compared to right TLE patients with a less clear lateralization pattern for comprehension tasks (Barr et al., 1990; Drane et al., 2013; Giovagnoli et al., 2005; Lah et al., 2004; Lambon Ralph et al., 2012; Voltzenlogel et al., 2006, 2015). Taking into account these neuropsychological data as well as the large meta-analysis on the neuroimaging data (Rice et al., 2015), this suggests that both verbal naming and comprehension tasks are left lateralised with a more dominant lateralisation effect for naming. 


\subsection{The present study}

The current study will investigate semantic problems in TLE patients with left or right ATL resection. The hypothesis is that left TLE patients perform particularly poorly on verbal semantic tasks, whereas the performance of right TLE patients is generally intact. This would be in agreement with a left lateralisation of verbal semantic information within the bilateral semantic system.

Semantic problems in TLE patients are often overlooked on standard semantic tests, but can be revealed by probing the meanings of low frequency words (Lambon Ralph et al., 2012). Therefore, the current study investigated this frequency effect in more detail by including multiple frequency levels. Moreover, hemispheric differences are likely to be overlooked when restricting to tasks on high frequency words, making it essential to include low frequent words to investigate the full impairment pattern of TLE patients. In more detail, we hypothesise that left TLE patients will perform poorly on low frequency verbal concepts, whereas performance will improve for higher frequency items.

In addition, as described in the previous section, when considering hemispheric differences associated with verbal semantic information, we need to distinguish between verbal expression and comprehension. Although both verbal expression and comprehension seem to be left lateralised, this lateralisation effect is stronger for expressive compared to comprehension tasks (see previous section). However, the use of low frequency concepts will aid in uncovering subtle hemispheric differences during semantic comprehension. Consequently, the current study included both a verbal expression and comprehension task. First, verbal expression was tested with two picture naming tasks. One of these tasks included pictures referring to high frequency concepts at a basic level, whereas the pictures of the second task referred to low frequency, subordinate concepts with low typicality (Garrard, Lambon Ralph, Hodges, \& Patterson, 2001). In other words, these items referred to specific concepts with distinctive features such as wooden clogs, cufflinks etc. We expect that this latter test will be especially sensitive to semantic impairments in left TLE patients, even at the individual level. Second, verbal comprehension was tested with the Synonym Judgement Task, which varies both word frequency and imageability (Lambon Ralph et al., 2012; Pobric et al., 2007). During this task, a probe word is presented at the top with three alternatives at the bottom and participants have to decide which of the bottom 
words is closest in meaning to the top word. Furthermore, the Synonym Judgement Task was paired with a non-semantic number task, which allows researchers to exclude non-semantic factors that can influence the results (Lambon Ralph et al., 2012; Pobric et al., 2007). Overall, we hypothesise that left TLE patients are more impaired than right TLE patients during verbal expression and comprehension, in agreement with a left lateralisation of verbal semantic information. Furthermore, this hemispheric difference will be more evident on words with a lower frequency of use.

In addition, both the expressive and comprehension tasks probe semantic processing. The inclusion of two tasks that probe semantics is important as it assures that we are revealing semantic impairments: if decreased performance is caused by a central semantic impairement we would expect impaired patients to perform badly on both tasks.

To investigate the impairment profiles, we will compare the patients group with a control group. Recently, the importance of including a control group that is matched for age, education and IQ has been highlighted (Butler \& Zeman, 2008; Elliott, Isaac, \& Muhlert, 2014) and current studies on resected TLE patients (see above) have not matched for all three factors and should therefore be interpreted with care. Therefore, the current study will use an age, education and IQ-matched control group. This is especially critical for mild semantic impairments that are only revealed by sensitive tasks using low frequency specific concepts. When it comes to low frequency words, levels of verbal semantic knowledge vary a lot with educational level and age, and this will influence the results.

The outcome of this study advances basic neuroscience as it reveals more about the role of the left and right ATL in semantic processing. This knowledge in turn can be used to develop clinical studies that further investigate clinical implications of semantic impairments in TLE patients. 


\section{Methods}

\subsection{Participants}

Fifty-eight participants were included in the current study. All participants were tested by an experienced neuropsychologist and the study was approved by the ethical committees of the University Jaume I, Castellón, and of the Hospital de la Fe, Valencia, Spain.

\subsection{Demographic patient data}

Thirty-two patients $(N=17$ male; $N=31$ right -handed) were recruited retrospectively from the Refractory Epilepsy Unit at the Hospital Universitario y Politécnico La Fe (Valencia, Spain). A Consort Flow Diagram of the patient selection is presented in Figure 1. Inclusion criteria for patients were: age range 18-65 years-old, diagnosis of refractory TLE with hippocampal sclerosis treated with standard antero-temporal lobectomy with amygdalohippocampectomy $(\mathrm{ATL}+\mathrm{AH})$, surgery performed $>6$ months before the test and patients with Spanish as their native language. Exclusion criteria were: active psychiatric condition, developmental delay, patients who underwent other neurosurgery for any reason including stereoelectroencehalography and presence of brain lesions (acute or chronic) at the time of the study. Furthermore, patients were excluded from the study when medicated with Topiramate (TPM) or Zonisamide (ZNS) as these antiepileptic drugs cause language impairments (Ojemann et al., 2001). All patients underwent standard antero-temporal lobectomy with amygdalohippocampectomy (ATL $+\mathrm{AH}), 17(N=8$ male, $N=16$ right-handed) in the left hemisphere and 15 ( $N=9$ male, $N=15$ right-handed $)$ in the right one, after being diagnosed with mesial temporal lobe epilepsy due to hippocampal sclerosis. The lateral extent of the temporal lobe resections was $4.5 \mathrm{~cm}$ from the temporal tip in the dominant hemisphere and $5.5 \mathrm{~cm}$ in non-dominant hemispheres. Resections were performed preserving the superior temporal gyrus (STG). Mean age at epilepsy onset was 11.4 years (range 1-40) and surgery had been performed after 28.6 years (range 1-59) of diagnosis. At the time of our study, the mean age of the patients was 43.4 (range 20-65) and surgery had been performed 4.30 years before our assessment (range 0-9 years). Twenty-five patients were in the late post-operative phase (after 12 months) and seven patients in the early post-operative phase (between 6 and 12 months) (months postsurgery $M=51.6, S D=31.1$ ). Twenty patients were seizure-free (Engel class I), three had rare disabling seizures (Engel class II) and nine had worthwhile improvements 
(Engel class III) since the surgery. Independent samples t-test showed that age at diagnosis $(t(30)=0.20, p=0.85)$, age at surgery $(t(30)=0.91, p=0.37)$ and months post-surgery $(t(30)=0.62, p=0.54)$ did not differ significantly between left and right TLE patients (means and standard deviations are presented in Table 2). Individual patient data regarding epilepsy features are summarized in Table 1.

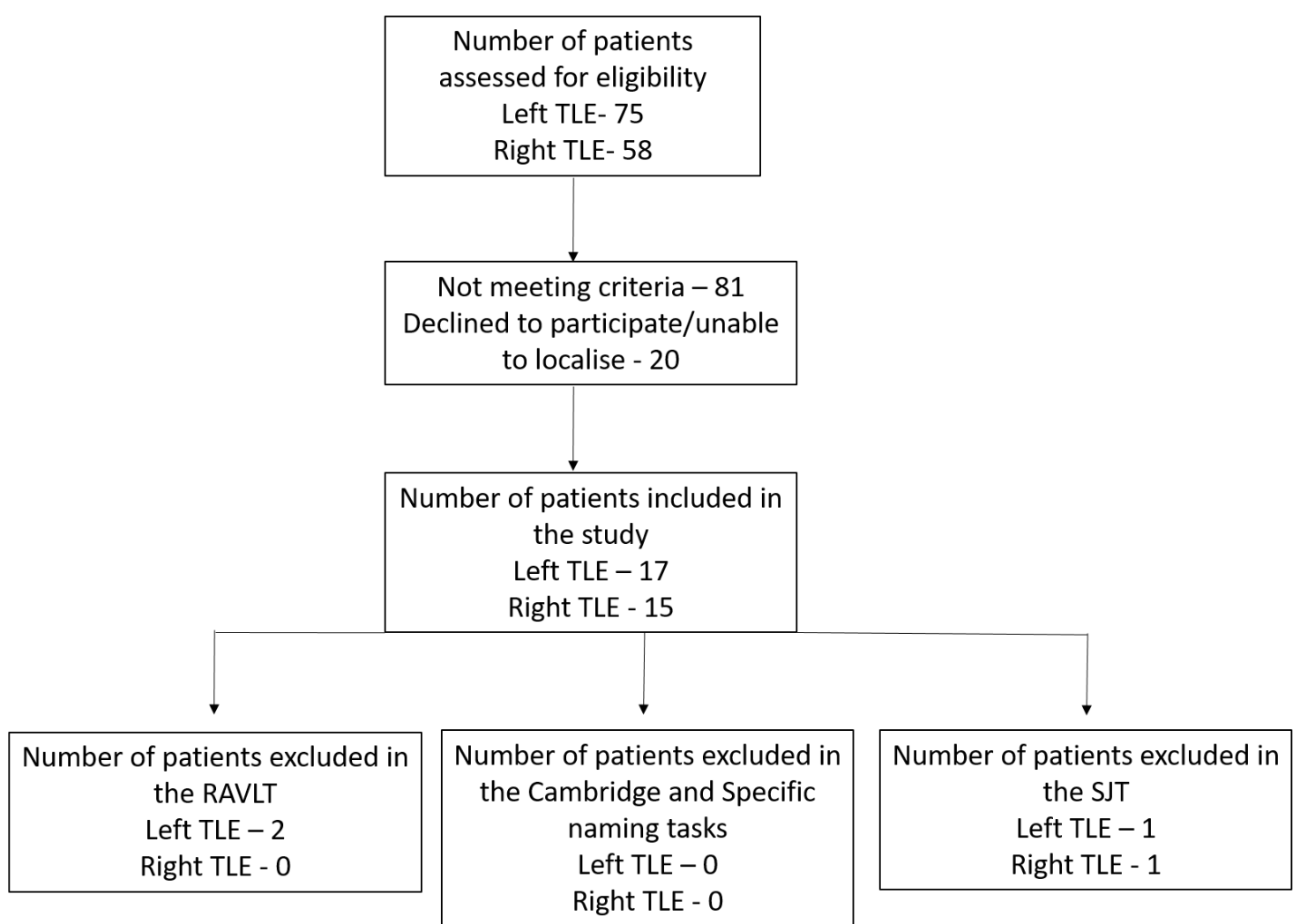

Figure 1. Consort Flow Diagram depicting patient selection.

\begin{tabular}{llllllll}
\hline Patient & Gender & Age & $\begin{array}{l}\text { Age at } \\
\text { onset }\end{array}$ & $\begin{array}{l}\text { Age at } \\
\text { surgery }\end{array}$ & $\begin{array}{l}\text { Side of } \\
\text { surgery }\end{array}$ & $\begin{array}{l}\text { Surgery } \\
\text { outcome* }\end{array}$ & Treatment** \\
\hline 1 & M & 37 & 1 & 28 & Right & I & OXC, CZP \\
2 & M & 59 & 14 & 58 & Right & I & LTG, VPA, LCM \\
3 & M & 62 & 5 & 58 & Right & I & LCM, CBZ \\
4 & F & 47 & 1 & 46 & Right & I & CZP, CBZ \\
5 & M & 39 & 3 & 32 & Right & I & CBZ \\
6 & F & 39 & 19 & 33 & Right & II & LEV, OXC, VPA \\
7 & F & 29 & 1 & 26 & Right & I & LEV, LCM, CBZ
\end{tabular}




\begin{tabular}{|c|c|c|c|c|c|c|c|}
\hline 8 & $\mathrm{M}$ & 54 & 14 & 49 & Right & II & LTG \\
\hline 9 & $\mathrm{M}$ & 50 & 18 & 42 & Right & III & CBZ, CLB \\
\hline 10 & M & 44 & 4 & 36 & Right & I & CBZ, LEV \\
\hline 11 & $\mathrm{M}$ & 50 & 18 & 49 & Right & I & LCM, VPA, LEV \\
\hline 12 & $\mathrm{~F}$ & 33 & 27 & 32 & Right & III & $\mathrm{CBZ}$ \\
\hline 13 & $\mathrm{~F}$ & 45 & 2 & 38 & Right & II & PB, LCM, LTG, CLB \\
\hline 14 & $\mathrm{~F}$ & 42 & 29 & 35 & Right & III & CBZ, LCM, BRV \\
\hline 15 & $\mathrm{M}$ & 47 & 12 & 46 & Right & I & OXC, LCM \\
\hline 16 & M & 20 & 5 & 16 & Left & III & LTG, LCM, PMP \\
\hline 17 & $\mathrm{M}$ & 34 & 1 & 30 & Left & I & LEV, LTG \\
\hline 18 & M & 65 & 5 & 64 & Left & I & OXC, LEV, LCM \\
\hline 19 & $\mathrm{~F}$ & 22 & 15 & 16 & Left & I & CBZ, CLB \\
\hline 20 & M & 34 & 1 & 27 & Left & I & VPA, LTG \\
\hline 21 & $\mathrm{~F}$ & 24 & 1 & 19 & Left & I & LTG \\
\hline 22 & M & 57 & 14 & 51 & Left & I & LCM, LTG, CLB \\
\hline 23 & $\mathrm{~F}$ & 49 & 1 & 45 & Left & III & CBZ, LEV, LCM \\
\hline 24 & M & 49 & 40 & 43 & Left & III & VPA, LEV, PHT \\
\hline 25 & $\mathrm{~F}$ & 58 & 17 & 54 & Left & I & LEV, LCM \\
\hline 26 & $\mathrm{~F}$ & 48 & 10 & 40 & Left & I & $\mathrm{CBZ}, \mathrm{CZP}$ \\
\hline 27 & $\mathrm{~F}$ & 34 & 19 & 29 & Left & III & CBZ, CLB \\
\hline 28 & $\mathrm{~F}$ & 30 & 11 & 29 & Left & I & VPA, LCM \\
\hline 29 & M & 59 & 8 & 56 & Left & III & LEV, LCM, CLB \\
\hline 30 & $\mathrm{~F}$ & 43 & 15 & 42 & Left & I & VPA, LCM \\
\hline 31 & $\mathrm{~F}$ & 30 & 9 & 25 & Left & III & CBZ, LCM, LEV, CLB \\
\hline 32 & M & 36 & 24 & 34 & Left & I & VPA, CLB, ESL \\
\hline
\end{tabular}

Table 1. Demographics information about the patients. M: male; F: female. Age in years. Engel classification*: I: free of disabling seizures; II: rare disabling seizures; III: worthwhile improvement of seizures; IV: no worthwhile improvement of seizures. Treatment**: OXC: oxcarbazepine; CZP: clonazepam; LTG: lamotrigine; VPA: valproic acid; LCM: lacosamide; CBZ: carbazepine; LEV: levetiracetam; CLB: clobazam; PB: phenobarbital; PMP: perampanel; PHT: phenytoin.

\begin{tabular}{ccccccc}
\hline & \multicolumn{2}{c}{ Age of diagnosis } & \multicolumn{2}{c}{ Age at surgery } & \multicolumn{2}{l}{ Months post-surgery } \\
\cline { 2 - 7 } & M & SD & M & SD & M & SD \\
\hline Left TLE & 11.76 & 10.21 & 36.47 & 14.45 & 48.29 & 27.55 \\
Right TLE & 11.07 & 9.71 & 40.53 & 10.15 & 55.47 & 37.30
\end{tabular}


Table 2. Means and standard deviations of the variables 'age of diagnosis' (in years), 'age at surgery' (in years) and 'months post-surgery'. Statistical analyses showed that left and right TLE patients did not differ significantly on these variables (see text).

\subsection{Age, education and IQ matched control group}

\begin{tabular}{lllllll}
\hline & \multicolumn{2}{l}{ Age } & \multicolumn{3}{c}{ Education } & \multicolumn{2}{c}{ Matrix Reasoning } \\
\cline { 2 - 7 } & $\mathrm{M}$ & $\mathrm{SD}$ & $\mathrm{M}$ & $\mathrm{SD}$ & $\mathrm{M}$ & $\mathrm{SD}$ \\
\hline Left TLE & 40.53 & 14.16 & 10.94 & 9.38 & 98.10 & 12.00 \\
Right TLE & 45.27 & 9.20 & 11.27 & 3.45 & 104.77 & 13.21 \\
Control & 40.62 & 7.77 & 10.69 & 2.74 & 105.34 & 10.13 \\
\hline
\end{tabular}

Table 3. Means and standard deviations of the variables 'age' (in years), 'education' (referring to years of education) and of the 'Matrix Reasoning Subtest'. Statistical analyses showed that left TLE, right TLE and control participants did not differ significantly on these variables (see text).

Control participants were recruited via local advertisements requesting participants between 18 and 50 years old without neurological or psychiatric impairments and without a university degree. From these advertisements, a pool of participants was gathered. Testing sessions took place after patient testing was finished, giving the opportunity to adjust the control group to the characteristics of the clinical groups on the variables 'gender', 'laterality' 'age' and 'years of education'. A total of 33 participants indicated they were interested in participating of which we choose 26 participants $(N=$ 13 male, $N=25$ right handed) based on an interview via the phone to match them to the patient groups. We tested at group level whether left TLE, right TLE and controls differed in age, gender and years of education, which was not the case (age: $F(2,55)=$ $1.14, p=0.33$; education: $F(2,55)=.16, p=0.85$; handedness $F(2,55)=.02, p=.98$; gender $X^{2}(2)=.93, p=.63$ ). Means and standard deviations are presented in Table 3 . This minimises the possibility that the results are influenced by any of these factors. Furthermore, we included the Matrix Reasoning subtest of non-verbal intelligence (a subtest of the Wechsler Adult Intelligence Scale III: WAIS III) to obtain the intellectual quotient (IQ) value based on non-verbal items. During this test of 26 items, the examinee looks at an incomplete matrix and identifies the missing section from one of 
five response options. The final IQ scores compare the performance of an individual with the average scores attained by members of that person's age group. An F-test between left TLE, right TLE and controls confirmed that scores on the Matrix Reasoning subtest did not differ between groups $(F(2,55)=1.96, p=.15$; see Table 3 for means and standard deviations).

In short, we carefully choose an education, age and intellectually (according to this scale) matched control group to avoid group differences to be caused by merely a difference in intelligence or education. We are thereby excluding an important bias that is commonly present in patient studies (Butler \& Zeman, 2008; Elliott et al., 2014). To our knowledge, this is the first study on semantic memory in resected TLE patients that matched for all three factors.

\subsection{Language and Memory battery}

The Language and Memory battery included five tests. First, we included the Rey Auditory Verbal Learning Test (RAVLT; Rey, 1958) which is an established neuropsychological test commonly used to examine learning as well as long-term memory impairments for verbal information. The learning phase consisted of 15 words that were read aloud on five consecutive trials. Each trial was followed by a free recall test in which participants were encouraged to repeat as many words as they could remember in any order. Learning ability was evaluated by the proportion of items learned on the fifth learning trial. After a 30-minute delay period, participants were required to recall once again as many words as they could remember in any order. Long-term memory problems were assessed by comparing the number of words recalled after 30 minutes with the number of words recalled on the fifth learning trial.

Second, participants performed two naming tests. The first one forms part of the Cambridge Semantic battery, which is used to test for semantic impairments in SD patients (Bozeat, Lambon Ralph, Patterson, Garrard, \& Hodges, 2000). It contains 64 black-and-white pictures. Most of these pictures refer to high frequency concepts at basic level. We will refer to this test as the Cambridge Naming Test. In addition, we added 40 coloured pictures (obtained from the internet), which referred to subordinate concepts with low typicality (Garrard et al., 2001). In other words, these items referred to specific concepts with distinctive features such as wooden clogs, cufflinks etc. We will refer to this test as the Specific Naming Test. The average word frequency of these 
two tests differed significantly $(t(97)=4.6 ; p<0.001$. Cambridge Naming Test: $M=$ 3.2, $S D=0.8$; Specific Naming Test: $M=2.5, S D=0.6$ : tested with the Espal database: www.bcbl.eu/databases/espal/). Figure 2 shows an example of the different items. Pictures of both naming tests were presented one-by-one on a computer and answers were recorded automatically in order to analyse accuracy and reaction time data. Both naming tasks began with three practice trials that were excluded from analyses. If it was clear that participants were unable to provide an answer, they were presented with a phonetic cue, which was the first letter of the word. If they still were not able to answer they were presented with the second and, if required, third letter of the word. This enabled us to record the influence of cueing on picture naming. Overall, this resulted in three variables of interest for analyses: (1) reaction time of correctly named items, (2) proportion of correctly named items out of all presented items and (3) proportion of correctly named items using a phonetic cue out of all presented items.

A) Cambridge Naming Test
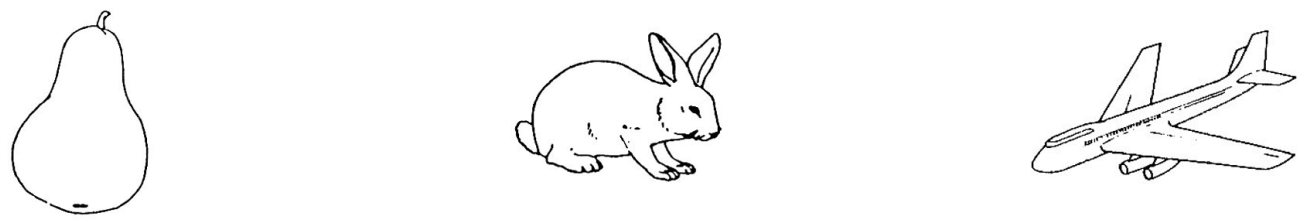

B) Specific Naming Test
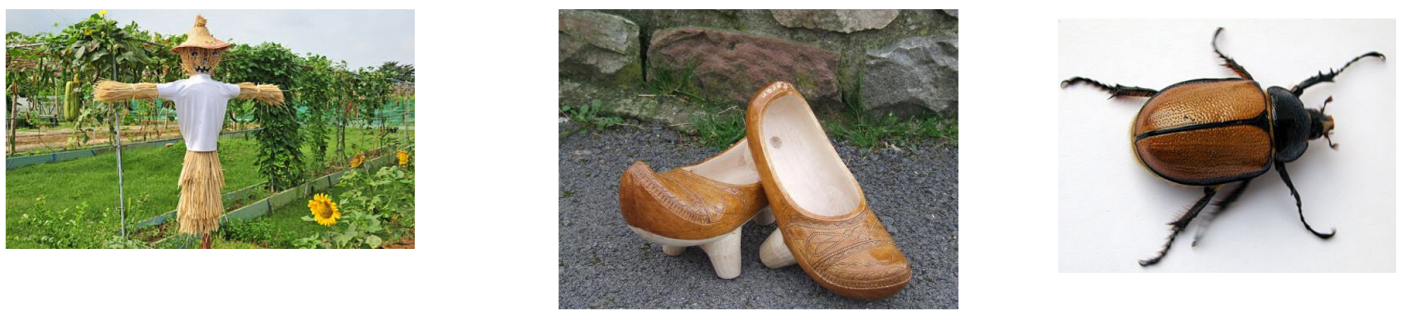

Figure 2. Examples of the expressive tasks. A) The Cambridge Naming Test forms part of the Cambridge semantic battery. B) The Specific Naming Test included pictures referring to low frequency, subordinate concepts with low typicality.

Third, participants performed the Synonym Judgement Task (Jefferies, Patterson, Jones, \& Lambon Ralph, 2009). A probe word is presented at the top of the screen with three alternatives at the bottom and participants have to decide which of the bottom words is closest in meaning to the top word. The original version was a $2 \times 3$ design varying word frequency (high versus low) and imageability (high, medium, 
low) orthogonally (with 16 trials in each condition), resulting in 96 trials. However, we used a Spanish translation of the test, made available by Sierpowska et al. (2015). This version used a $2 \times 2$ design including two levels of imageability and frequency (high and low), resulting in 64 trials. Sierpowska et al. (2015) obtained imageability values from a set of 25 healthy participants using a 7-point Likert scale. The average imageability values of the trials differed significantly between the high and low condition $(t(62)=$ 36.89, $p<0.001$. High Imageability Condition: $M=261.7, S D=41.8$; Low Imageability Condition: $M=623.9, S D=36.6)$. The average word frequency in the low and high frequency condition also differed significantly $(t(62)=20.07, p<0.001$. High Frequency Condition: $M=4.28, S D=0.24$; Low Frequency Condition: $M=3.04, S D=$ 0.26; word frequency values obtained from the Espal database (www.bcbl.eu/databases/espal/). In addition, the two word frequency conditions were matched for imageability $(t(62)=0.66 ; p=.51 ; M=437.85, S D=183.73$ for low frequency trials and $M=447.80, S D=192.34$ for high frequency trials) and vice versa $(t(62)=0.21, p=.83 ; M=3.72, S D=0.66$ for low imageability trials and $M=3.61, S D$ $=0.70$ for high imageability trials). The test was preceded by eight practice trials that were not included in the analyses. Accuracy and reaction time data were measured for the four conditions of the $2_{\text {(frequency) }} 2_{\text {(imageability) }}$ design. Accuracy was computed as the proportion correctly responded items of each condition and reaction time data only included correctly named items.

Finally, the Synonym Judgement Task is paired with a non-semantic task on double digit numbers using the same format as the semantic task (i.e., one item at the top and three at the bottom; Lambon Ralph et al., 2012; Pobric, Jefferies, \& Ralph, 2007). Participants have to decide which of the bottom numbers was closest in value to the top item. This allowed us to test general non-semantic impairments. As with the Synonym Judgement Task, the test was preceded by eight practice trials that were not included in the analyses. Accuracy was computed as the proportion correctly responded items and reaction time data only included correctly answered items.

The two naming tests and the Synonym Judgement Task were performed on a laptop. These tasks were presented in E-prime (www.pstnet.com) and analysed using Matlab (www.mathworks.com) and SPSS (www.ibm.com). 


\subsection{Procedure}

All participants were tested by an experienced neuropsychologist. After a preliminary interview, participants performed the following tests in a single session: (1) The RAVLT. (2) During the 30 minute delay of the RAVLT, the Matrix Reasoning subtest of non-verbal intelligence. This assures that participants cannot use rehearsal techniques in the RAVLT, which leads to confounding results (Elliott et al., 2014); (3) the Cambridge Naming Test; (4) the Specific Naming Test; and (5) The Synonym Judgement Task followed by the (6) number task.

\subsection{Statistical analyses}

First, we ran Kolmogorov-Smirnov tests on the Rey Auditory Verbal Learning Test, Cambridge Naming Test, Specific Naming Test and Synonym Judgement Task to investigate distribution of scores. Group was included as a factor. Both the left and right TLE patient groups showed a normal distribution $(p>0.05)$ on all tests. However, the control group showed a deviant distribution on all four tasks. However Ghasemi and Zahediasl (2012) suggested that with large sample sizes, the violation of normality should not cause major problems. Therefore, as we had a relatively large control group $(N=26)$, we decided to continue with parametric tests.

Second, we tested the performance on the RAVLT. Separate one-way ANOVAs were used to test for group differences between left TLE, right TLE and control participants with respect to learning ability (tested on the fifth learning trial) as well as for long-term memory problems (tested by comparing the 30 minute delayed recall trial with the fifth learning trial).

Third, two one-way ANOVAs were used to test for group differences on both the Cambridge and Specific Naming Test. The accuracy data excluded items which needed cueing, as cueing was only used when it was clear the participant could not name the item. In addition, group differences on the effectiveness of cueing was tested by the proportion of successfully named items after cueing compared to all included items, irrespective of whether 1, 2 or 3 cue letters were required.

Furthermore, we looked at the word frequency effect in more detail. To do this we combined the pictures of both the Cambridge and Specific Naming Test and categorised the pictures in four groups of 26 items (i.e., low frequency, medium low, 
medium high and high frequency), using the log count word frequency values of the Espal database (http://www.bcbl.eu/databases/espal/). This enabled us to inspect performance at four different word frequency levels in the patient and control groups. As explained in the Introduction, we expected an improvement in performance with increasing word frequency. Group differences were investigated by using a within subject contrast in a $3_{\text {(group) }} \times 4_{\text {(word frequency) }}$ mixed ANOVA to examine the presence of a linear group*word frequency interaction. Planned comparisons compared differences in linear increase between each group pair.

Fourth, we performed a 3 (group) $x 2_{\text {(frequency) }} 2_{\text {(imageability) }}$ mixed-model ANOVA to study the Synonym Judgement Task. Planned comparisons compared the difference in frequency effect between each group pair. Similarly, the difference for low and high imageability items were tested for each group pair.

Fifth, reaction time data was tested with separate one-way ANOVAs for the Specific Naming Test, Cambridge Naming Test, Synonym Judgement Task and the number task. Furthermore, for the Synonym Judgement Task we included a Task $(2) \mathrm{X}$ Group $_{(3)}$ mixed ANOVA, including the number task.

Finally, we ran Pearson's correlation analyses to test for correlations between the different tasks included in the study. In addition, we included the demographics 'age', 'age at diagnosis', and 'months post-surgery'.

\section{Results}

\subsection{Rey Auditory Verbal Learning Test (RAVLT)}

After the fifth repetition of the word list, significant group differences were found on the proportion of learned words $(F(2,55)=12.74, p<0.001 ; M=0.63, S D=0.17$ for left TLE; $M=0.69, S D=0.16$ for right TLE; $M=0.86, S D=0.13$ for controls). Posthoc Bonferroni tests showed that both left and right TLE patients performed significantly worse than control participants during the learning phase of the RAVLT test ( $p<0.001$ and $p=0.003$, respectively). Furthermore, significant group differences were found for long-term memory after the delay of 30 minutes $(F(2,55)=5.64, p=$ $0.006 ; M=0.60, S D=0.33$ for left TLE; $M=0.80, S D=0.13$ for right TLE; $M=0.81$, 
$S D=0.13$ for controls). Post-hoc Bonferroni tests showed that left TLE patients performed significantly worse compared to right TLE patients and controls $(p=0.034$ and $p=0.007$, respectively). In addition, right TLE patients were not significantly impaired compared to controls $(p>0.99)$.

\subsection{Picture naming}

Separate one-way ANOVAs showed significant group differences on both the Cambridge Naming Test $(F(2,55)=8.91, p<0.001)$ and the Specific Naming Test $(F(2,55)=28.89, p<0.001)$, which is illustrated in Figure 3. Post-hoc Bonferroni tests showed that left TLE patients performed significantly worse than right TLE patients and controls on both naming tests $(p<0.007)$. In contrast, right TLE patients did not perform worse than controls $(p>0.30)$.

Furthermore, we examined the influence of phonetic cues. Group differences were significant on the Cambridge Naming Test $(F(2,55)=5.30, p=0.008 ; M=0.10$, $S D=0.10$ for left TLE; $M=0.04, S D=0.04$ for right TLE; $M=0.03, S D=0.02$ for controls) but not on the Specific Naming Test $(F(2,55)=2.71, p=0.08 ; M=0.20, S D=$ 0.10 for left TLE; $M=0.17, S D=0.11$ for right TLE; $M=0.14, S D=0.07$ for controls). Post-hoc Bonferroni tests showed that the improvement on the Cambridge Naming Task following cueing was caused by a significant improvement in performance in the left TLE patient group compared to right TLE patients and controls $(p=0.019$ and $p=$ 0.001 , respectively).

Moreover, we looked at the individual performance on these two naming test (see Figure 4). This showed that the left TLE patients, with the exception of three, performed over two standard deviations below average on the Specific Naming Test, making this test sensitive at the individual level. In contrast, as can be seen in Figure 3, this sensitivity at the individual level is not achieved by the Cambridge Naming Test. 


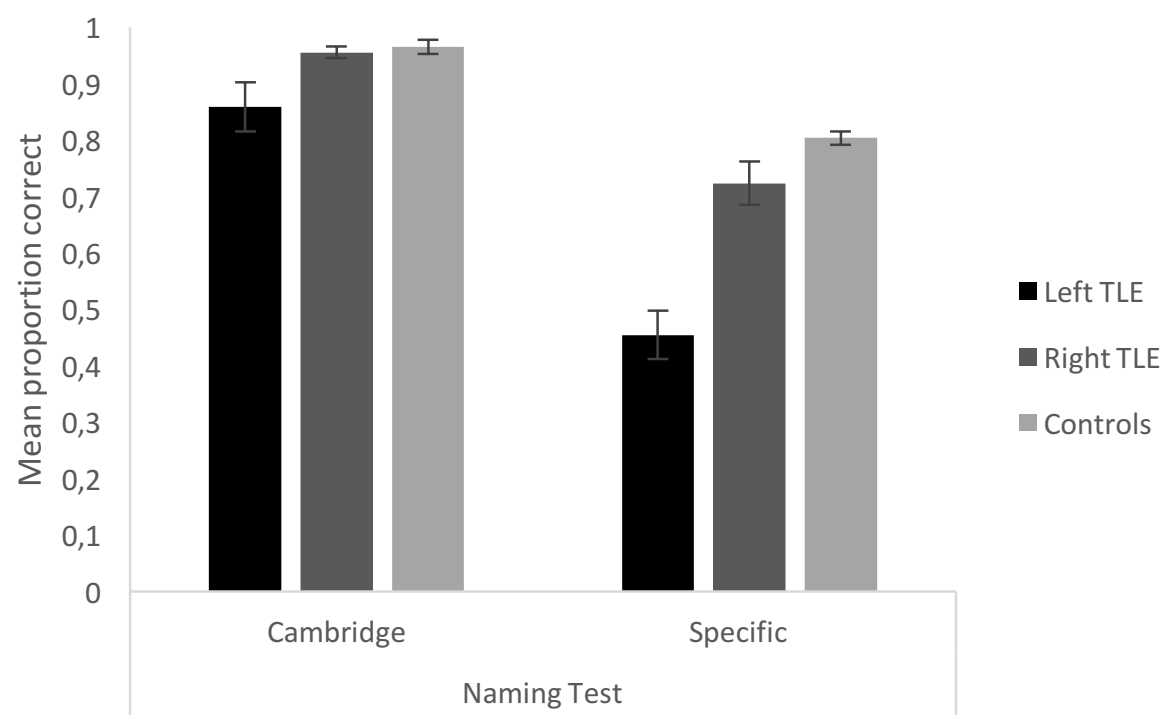

Figure 3. Mean proportion correct on the Cambridge and Specific Naming Test. Significant group differences were found on both tests. Post-hoc Bonferroni tests showed that left TLE patients performed significantly worse than right TLE patients and controls on both naming tests. Error bars present the standard error mean.

\section{Cambridge Naming Test}

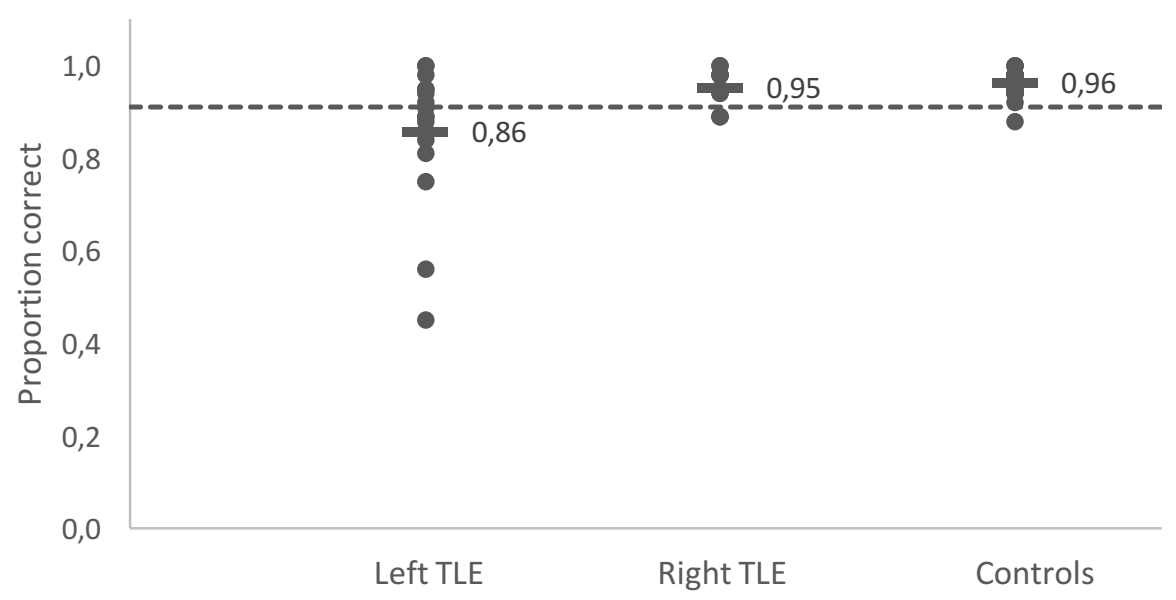




\section{Specific Naming Test}

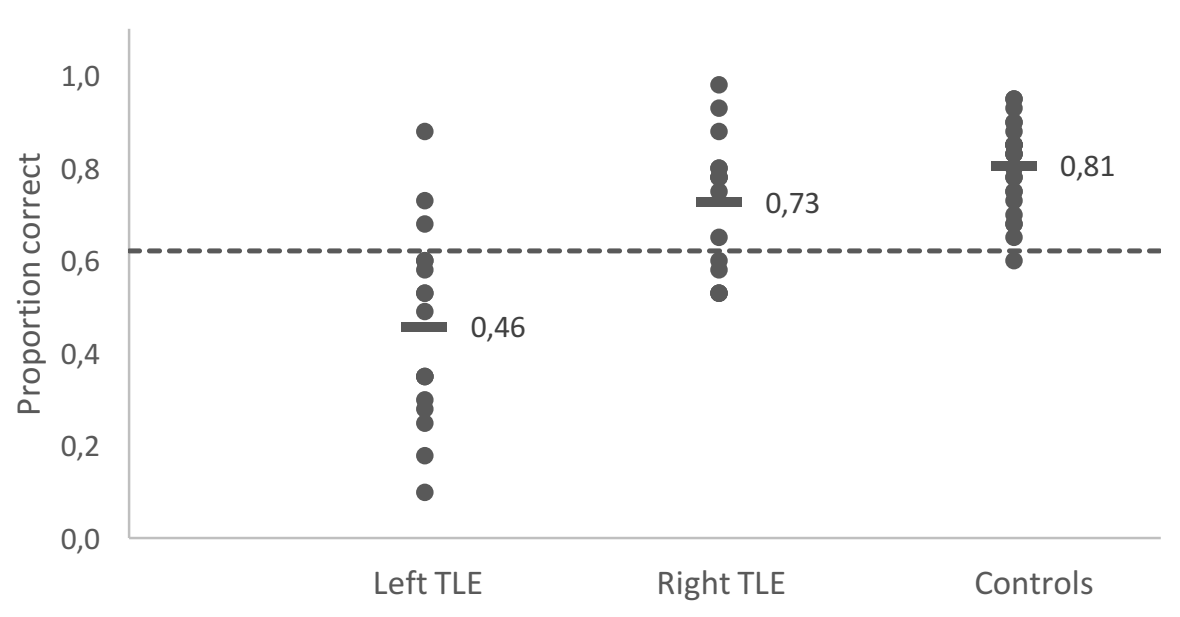

Figure 4. Individual results on the Cambridge and Specific Naming Test, separated by the three groups. Average group values are labelled. The dashed line marks two standard deviations below the performance of the control group. This shows that a large part of the left TLE patients show a semantic impairment on the Specific Naming Test.

Finally, as explained in Statistical Analyses section 2.5, we combined the pictures of both naming tasks and categorised them into four groups (i.e., low, medium low, medium high and high frequent). The $3_{\text {(group) }} \times 4_{\text {(word frequency) }}$ mixed ANOVA showed a significant main effect of Group $(F(2,55)=21.27, p<0.001)$ caused by poorer performance of the left TLE group compared to the other two groups. In addition, a main effect of Word Frequency $(F(3,165)=158.50, p<0.001)$ was caused by poorer performance on low compared to high frequency words. Further inspection revealed group differences on all four frequencies $(F(2,55)=34.97, p<0.001, F(2,55)=16.19, p$ $<0.001, F(2,55)=12.06, p<0.001, F(2,55)=4.68, p=0.013$ from low to high). Posthoc Bonferroni tests showed that left TLE patients differed on all four levels from controls and on the first three levels from right TLE patients (but not on the fourth level, $p=0.11)$. Right TLE patients did not differ from control participants on any of the four levels $(p>0.50)$.

Both main effects were driven by the significant Group x Word Frequency interaction $(F(2,55)=38.58, p<0.001)$. Planned comparisons revealed a steeper increase for word frequency for the left TLE patient group compared to right TLE patients $(p<0.001)$ and controls $(p<0.001)$. In contrast, this word frequency effect was 
not significant when comparing right TLE patients and controls $(\mathrm{p}=0.256)$. Results are presented in Figure 5.

Overall, the results show that naming difficulties are particularly visible on low frequency objects, whereas performance increases with increasing word frequency. In addition, only left TLE patients seem to suffer from naming problems.

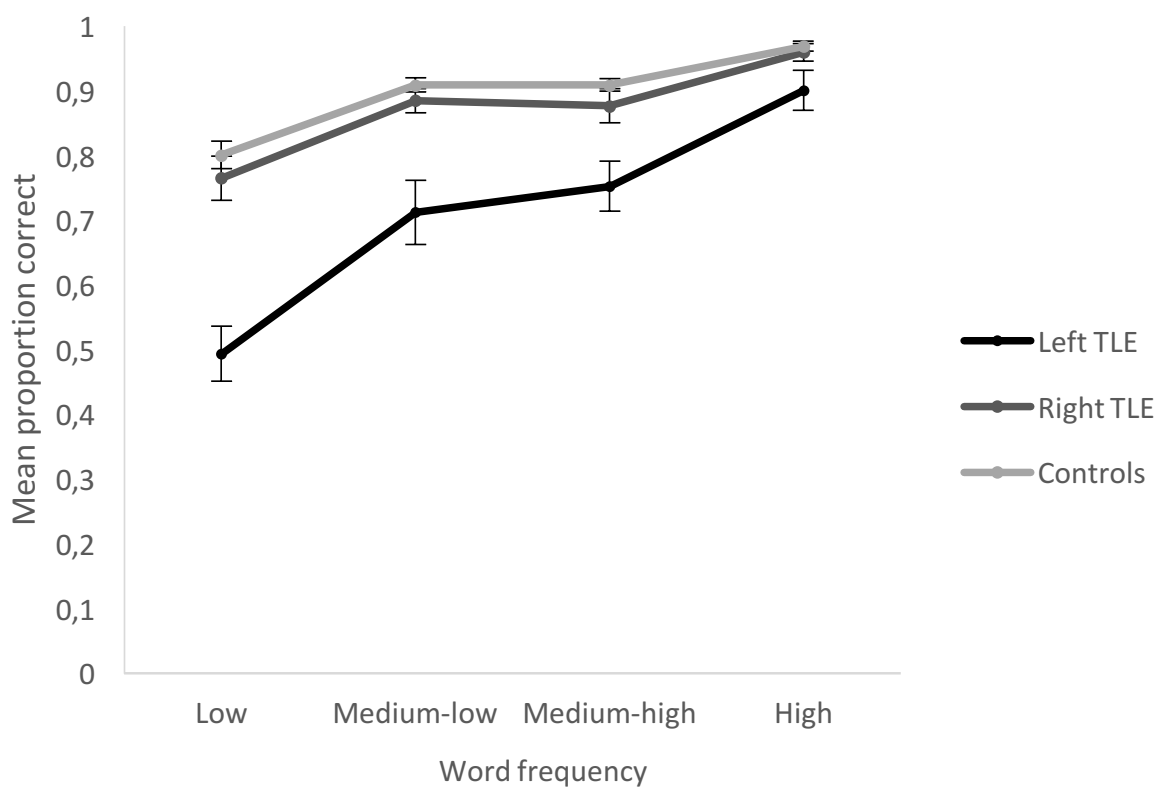

Figure 5. Naming results for the different word frequency levels. The items of the Cambridge and Specific Naming Tests were combined and grouped according to word frequency. This shows that the naming impairment of left TLE patients is dominantly apparent on low frequent items. Error bars present the standard error mean.

\subsection{Synonym judgement task (SJT)}

We performed a $3_{\text {group }} \times 2_{\text {frequency }} \times 2_{\text {imageability }}$ mixed ANOVA. Results are presented in Figure 6. Results showed a main effect of $\operatorname{Group}(F(1,53)=7.46, p=0.001)$, caused by impaired performance of left TLE patients compared with controls. Right TLE patients did not differ from left TLE patients or controls $(p<0.15)$. Furthermore, there was a main effect of Word Frequency $(F(1,53)=5.32, p=0.025)$ and Imageability $(F(1,53)=$ $61.17, p<0.001)$. The $3_{\text {group }} \times 2_{\text {frequency }} \times 2_{\text {imageability }}$ interaction was not significant $(F(2,53)=.68, p=0.51)$. However, there was a significant Group x Word Frequency 
interaction $(F(2,53)=4.35, p=0.018)$ and a Group $\mathrm{x}$ Imageability interaction $(F(2,53)$ $=4.25, p=0.019$ ) Planned comparison revealed a greater difference between low and high frequency words for the left TLE group compared to controls $(p<0.001)$ and the right TLE group $(p<0.05)$. In other words, the frequency effect was stronger for left TLE patients compared to the right TLE and control group. Similarly, planned comparisons showed that the difference between low and high imageability words was greater for the left TLE group compared to controls $(p<.001)$ and the right TLE group $(p<0.05)$.

Finally, the Synonym Judgement Task included the non-semantic control task to test for more general, non-semantic deficits. A one-way ANOVA revealed significant group differences on the number task $(F(53)=3.67, p=0.03, M=0.89, S D=0.03$ for left TLE; $M=0.86, S D=0.08$ for right TLE; $M=0.90, S D=0.03$ for controls). Posthoc Bonferroni analyses showed that right TLE patients performed significantly worse compared to controls $(p=0.03)$, but not compared to left TLE patients $(p=0.24)$. In addition, left TLE patients did not differ significantly from controls $(p=1.00)$. This indicates that the right TLE patients presented with general non-semantic deficits but the left TLE patients did not.

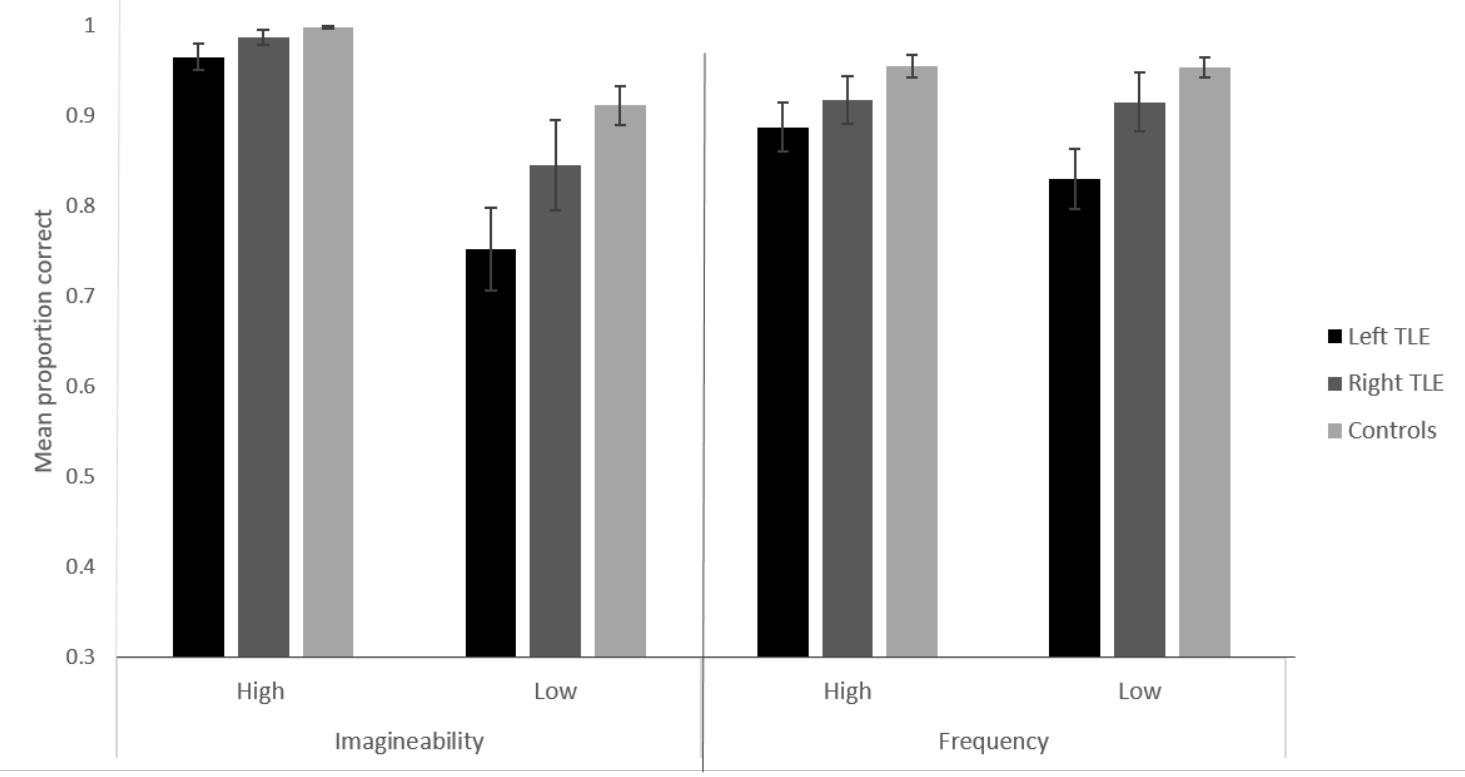

Figure 6. Synonym Judgement Word Frequency x Imageability analysis. This showed a Group x Word Frequency as well as a Group x Imageability interaction, caused by a significant difference between left TLE patients with controls. Error bars denote standard error mean. 


\subsection{Reaction time data}

We analysed the reaction time (RT) data of the included tasks (on correct trials only). Separate $\mathrm{F}$ tests for each task showed significant group differences on the: Cambridge Naming Test $(F(2,50)=10.60, p<0.001)$, Specific Naming Test $(F(2,50)=21.81, p<$ $0.001)$ Synonym Judgement Task $(F(2,55)=8.73, p=0.001)$ and the number task $(F(2$, $55)=7.17, p=0.002$ ). Post-hoc Bonferroni test showed that left TLE patients showed a general slowing on all tasks compared to controls. In contrast, right TLE patients only showed decreased RTs on the Specific Naming Task compared to controls $(p>0.10$ for the remaining tests).

The analyses of RTs for the Synonym Judgement Task was conducted using a $\operatorname{Task}_{(2)} \times \operatorname{Group}_{(3)}$ mixed ANOVA, including the number task. This showed a significant main effect of group $(F(2,53)=9.35, p<0.001)$, task $(F(1,53)=64.57, p<0.001)$ and a significant interaction $(F(2,53)=4.10, p=0.022)$. Planned comparisons between the three groups revealed poorer performance of left TLE patients compared to controls ( $p$ $<0.001$ ) on the semantic compared to the number task. In addition, performance did not differ for right TLE patients compared to controls $(p=0.50)$ and approached significance when compared with the left TLE patient group ( $p=0.06)$.

Overall, this pattern of increased RTs seems to indicate a semantic impairment for left but not right TLE patients on the Synonym Judgement Task. Left TLE patients were impaired on the Synonym Judgement Task and significantly more so than on the number task, excluding general slowing down as a factor. In contrast, right TLE patients did not differ from controls on the Synonym Judgement Task. With respect to the Specific Naming Task, both left and right TLE patients showed increased reaction times. However, we cannot examine whether this is specific to a semantic impairment or is caused by a general reduction in processing speed, as we do not have a comparative non-semantic task. 


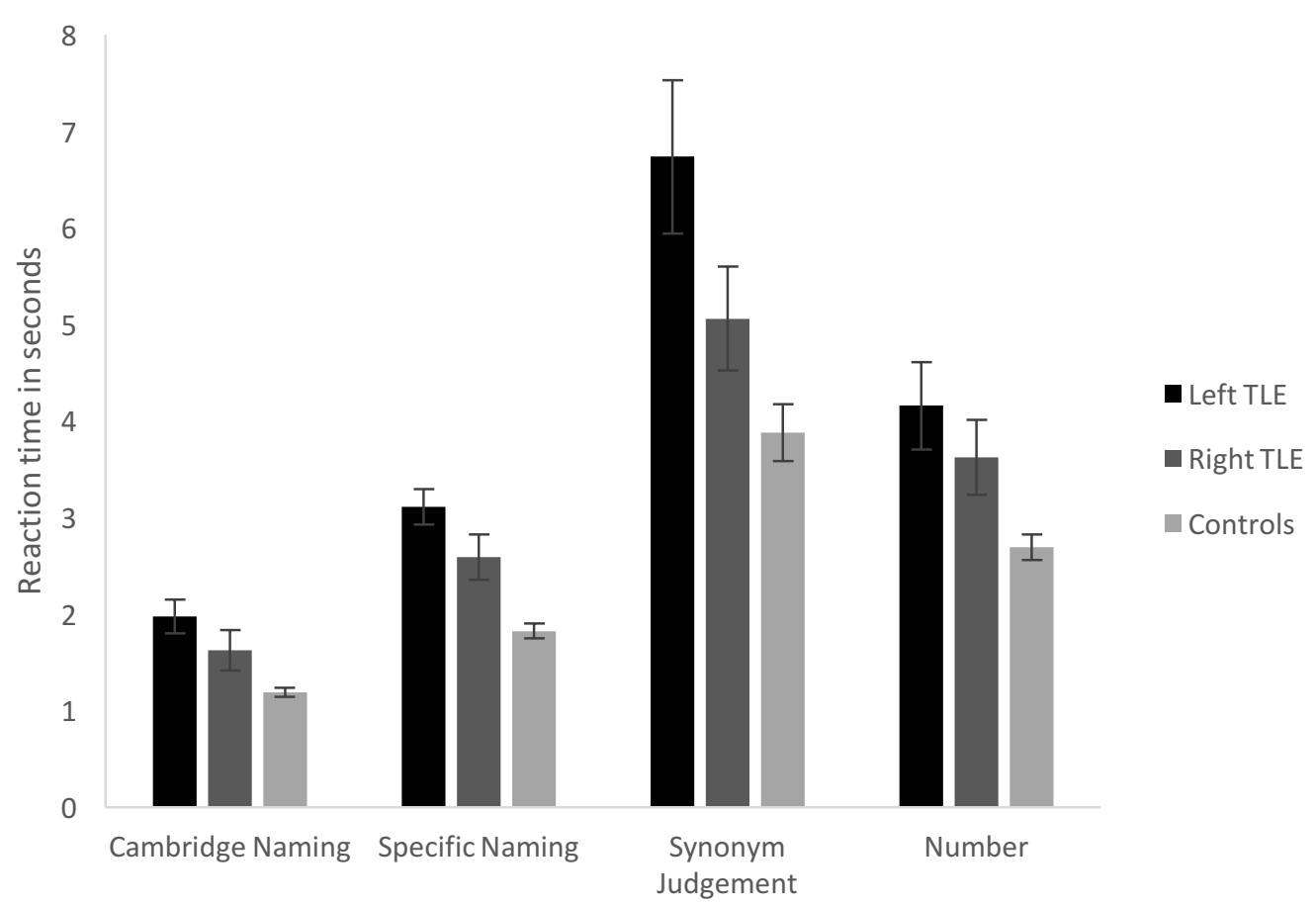

Figure 7. Reaction time data of all semantic tasks. A decrease in reaction time was found for left TLE patients on all tasks. In addition, these patients showed a significant difference between the Synonym Judgement Task and the number task, indicating a semantic impairment and not a general slowing down of cognitive processing. Error bars present the standard error mean.

\subsection{Correlation analyses}

Both the picture naming and semantic association require semantic processing.

Therefore, semantic impairments should be apparent in the Cambridge Naming Test, the Specific Naming Test and the Synonym Judgement task and these measures should be correlated. As described in the previous section, performance on these tasks was impaired in left TLE patients. Moreover, Pearson's correlation analyses showed that the results on these tasks were correlated in left TLE patients, in agreement with our expectations (see Table 4). Furthermore, age of diagnosis was negatively correlated with the Synonym Judgement Task in left TLE patients and with delayed recall in right TLE patients.

With respect to the RAVLT, we looked at both the fifth trial of immediate recall as well as 30 minutes delayed recall. Immediate recall was negatively correlated with age for all three groups, which means that younger participants perform better on this task. In contrast, the 30 minute delayed recall task was negatively correlated with age but only for the left TLE patients. Furthermore, this task was positively correlated with the Cambridge Naming Test for left TLE patients, suggesting that both tasks might 
share some common factor. Finally, for left TLE patients, months post-surgery correlated positively with the Cambridge and Specific Naming task as well as with immediate recall.

\begin{tabular}{|c|c|c|c|c|c|c|c|c|c|c|}
\hline & Variables & 1 & 2 & 3 & 4 & 5 & 6 & 7 & 8 & 9 \\
\hline \multirow[t]{4}{*}{1} & Age & & & & & & & & & \\
\hline & Left TLE & - & & & & & & & & \\
\hline & Right TLE & - & & & & & & & & \\
\hline & Controls & - & & & & & & & & \\
\hline \multirow[t]{4}{*}{2} & Age of diagnosis & & & & & & & & & \\
\hline & Left TLE & .12 & - & & & & & & & \\
\hline & Right TLE & -.00 & - & & & & & & & \\
\hline & Controls & - & - & & & & & & & \\
\hline \multirow[t]{4}{*}{3} & Months post-surgery & & & & & & & & & \\
\hline & Left TLE & -.13 & -.02 & - & & & & & & \\
\hline & Right TLE & -.11 & -.20 & - & & & & & & \\
\hline & Controls & - & - & - & & & & & & \\
\hline \multirow[t]{4}{*}{4} & Cambridge naming & & & & & & & & & \\
\hline & Left TLE & -.30 & -.24 & $.74 * *$ & - & & & & & \\
\hline & Right TLE & -.16 & -.25 & .08 & - & & & & & \\
\hline & Controls & -.03 & - & - & - & & & & & \\
\hline \multirow[t]{4}{*}{5} & Specific naming & & & & & & & & & \\
\hline & Left TLE & -.25 & -.35 & $.64 * *$ & $.79 * *$ & - & & & & \\
\hline & Right TLE & -.04 & .05 & -.04 & $.56^{*}$ & - & & & & \\
\hline & Controls & .25 & - & - & $.66 * *$ & - & & & & \\
\hline \multirow[t]{4}{*}{6} & Synonym Judgement & & & & & & & & & \\
\hline & Left TLE & -.12 & $-.72 *$ & .38 & $.56 * *$ & $.72 * *$ & - & & & \\
\hline & Right TLE & $-.62 *$ & .40 & -.02 & -.08 & .41 & - & & & \\
\hline & Controls & .08 & - & - & -.03 & -.12 & - & & & \\
\hline \multirow[t]{4}{*}{7} & Immediate recall & & & & & & & & & \\
\hline & Left TLE & $-.53 *$ & -.01 & $.52 *$ & $.57 *$ & $.54 *$ & .22 & - & & \\
\hline & Right TLE & $-.63 *$ & -.10 & -.26 & .28 & .32 & .48 & - & & \\
\hline & Controls & $-.42 *$ & - & - & .29 & .17 & .25 & - & & \\
\hline \multirow[t]{4}{*}{8} & Delayed recall & & & & & & & & & \\
\hline & Left TLE & $-.61 *$ & -.20 & .22 & $.59 *$ & .45 & .28 & $.64 *$ & - & \\
\hline & Right TLE & .28 &.$- .60 *$ & .31 & .49 & .40 & -.12 & -.07 & - & \\
\hline & Controls & -.08 & - & - & .13 & .08 & -.11 & .32 & - & \\
\hline \multirow[t]{4}{*}{9} & Number task & & & & & & & & & \\
\hline & Left TLE & -.26 & -.33 & .16 & -.01 & .13 & .50 & -.05 & -.04 & - \\
\hline & Right TLE & -.47 & -.12 & .06 & .083 & $.55^{*}$ & $.76^{* *}$ & .38 & -.02 & - \\
\hline & Controls & $-.39 *$ & - & - & .056 & .02 & -.06 & .18 & .25 & - \\
\hline
\end{tabular}

Table 4. Pearson correlation matrix on the task performance of the patients and control participants, including the patient demographic variables 'age of diagnosis' and 'months post-surgery'. Statistical significance $* p<0.05, * * p<0.01$

\section{Discussion}

The current study investigated hemispheric differences for verbal semantic memory in patients with epilepsy who underwent unilateral anterior temporal lobe (ATL) lobectomy. Left but not right TLE patients showed semantic impairments for verbal information, which were particularly apparent for low frequency objects. The same pattern was seen for both the expressive naming task and the Synonym Judgement comprehension task. The outcome of the present study has implications not only for 
current models on semantic memory but also for clinical management of epilepsy patients, which will be discussed in the next sections.

\subsection{Lateralization in the semantic system}

The current results provide insight into the lateralization effects suggested by newer versions of the hub-and-spoke's model (Rice, Hoffman, et al., 2015; Rice, Lambon Ralph, et al., 2015; Schapiro et al., 2013). Neuroimaging results indicate that the semantic system in the ATL is bilateral, with a graded specialization depending on connections with modality-specific regions (Binney, Parker, \& Lambon Ralph, 2012; Rice, Caswell, Moore, Hoffman, \& Lambon Ralph, 2018; Rice, Hoffman, \& Lambon Ralph, 2015; Rice, Lambon Ralph, \& Hoffman, 2015; Visser \& Lambon Ralph, 2011). For example, during verbal tasks, the role of the left ATL is more dominant due to connections with the left hemisphere language network. In addition, the model states that such a bilateral system is robust against unilateral damage as the remaining ATL can take over the function of the impaired hemisphere (Schapiro et al., 2013). However, this further depends on the type of task: (a) if a function is bilaterally supported (i.e., general and non-verbal semantics) then the system is quite robust to unilateral damage and shows better recovery; but (b) where a function is more lateralised (cf. naming) there is less robustness to damage and also less opportunity for recovery (Schapiro et al., 2013). Nevertheless, until now interpretations of the model suggested that damage to one of the ATLs should always lead to some degree of semantic impairment, irrespective of modality (Rice, Hoffman, et al., 2015; Rice, Lambon Ralph, et al., 2015) and the current results contradict this.

We suggest that the absence of semantic impairments in right TLE patients can be explained by two complementing mechanisms. First, verbal processing is so left lateralised that (even though actively involved in the healthy brain) the right ATL becomes dispensable. Second, the bilateral ATL uses a compensatory system, in which damage to the one unilateral ATL leads to an upregulation in the remaining ATL. Evidence of such a compensatory system comes from two combined rTMS-fMRI studies, in which rTMS to the unilateral ATL led to an upregulation in the contralateral ATL and increased inter-hemispheric ATL interaction, (note that this was only tested with left ATL rTMS; Binney \& Lambon Ralph, 2015; Jung \& Lambon Ralph, 2016). Similar upregulatory systems are also found outside the language system (O'Shea, 
Sebastian, Boorman, Johansen-Berg, \& Rushworth, 2007). Although such an upregulation most likely exists after either left or right ATL lobectomy, it will not be sufficient to overcome all language impairments in the case of left ATL damage, due to the strong language lateralisation. In contrast, such an upregulatory system can aid in overcoming the mild language impairments caused by right ATL damage.

To sum up, we suggest that a left hemisphere language lateralization in combination with a compensatory mechanisms enables the semantic system to function well on verbal tasks after right ATL damage. Future research is required to investigate the details of such mechanisms further.

\subsection{Picture Naming}

The current study investigated hemispheric differences in TLE patients with ATL resection during verbal expression on two picture naming tasks: the Cambridge Naming Test and the Specific Naming Test. Whereas the majority of the items on the former test refer to high frequency concepts at the basic level, the latter test includes items referring to specific low word frequency items. This enabled us to inspect performance on both tests and, in addition, to combine both tests to vary frequency levels and investigate the frequency effect in more detail. As expected, left TLE patients performed poorly on low word frequency items and increasingly better on higher word frequency items. In contrast, this increased word frequency effect was absent in right TLE patients and controls. So even though naming problems are already visible on highly frequent objects in the current study as well as previous TLE studies (Bell et al., 2001; Davies et al., 1998; Gabrieli, Cohen, \& Corkin, 1988; Hamberger, 2015; Hermann, 2015; IvesDeliperi \& Butler, 2012; Lou Smith, Elliott, \& Lach, 2006), including varying frequency levels in semantic tasks can aid in forming a detailed clinical profile of a patient group. Furthermore, the Specific Naming Test was more likely to classify a patient as impaired. This is important for clinical purposes, where individual results are more critical than group results.

Previous TLE studies have shown such a left lateralisation for naming for resected and non-resected adults and children (Drane et al., 2013; Lah \& Smith, 2015; Lambon Ralph et al., 2012; Rice et al., 2018; Voltzenlogel et al., 2015). However, some naming studies on TLE patients without lobectomy did not find lateralisation effects 
(Giovagnoli et al., 2016b; Messas, Mansur, \& Castro, 2008; Seidenberg et al., 2002; Smith \& Lah, 2011). This pattern seems to indicate that operated patients show lateralisation effects on naming tasks but patients without lobectomy do not always.

These inconsistent lateralisation patterns could rely on various interacting study factors. First, as lateralisation effects are found in patients with ATL lobectomy, this seems to indicate that the amount of ATL damage is of importance. In other words, the extensive damage caused by left ATL lobectomy causes severe naming difficulties, making it easy to expose laterality effects. In line with this, a recent paediatric study found naming declined after unilateral ATL resection but only for left TLE patients, suggesting that severe damage to the left ATL causes naming impairments (Lah \& Smith, 2015). Second, patients with and without ATL lobectomy present with very different profiles regarding clinical seizures as well as subclinical epileptiform activity and this might be another confounding factor (Javidan, 2012; Semah et al., 1998).

Furthermore, with respect to cueing, the only group differences were found for the Cambridge Naming Test, which showed improved naming performance after cueing for the left TLE patients. This is most likely caused by the fact that right TLE and control participants performed near ceiling level making cueing unnecessary for them.

\subsection{Synonym Judgement Task}

We tested for hemispheric differences on a verbal comprehension task. Previous research on Semantic Dementia patients has shown that left lateralisation for verbal information is less clear for comprehension tasks Lambon Ralph et al.(2001). Similarly, a left lateralisation for verbal comprehension is less clear when examining the TLE literature (Barr, Goldberg, Wasserstein, \& Novelly, 1990; Drane et al., 2013; Lah, Grayson, Lee, \& Miller, 2004; Lambon Ralph, Ehsan, Baker, \& Rogers, 2012; Rice, Caswell, Moore, Hoffman, \& Lambon Ralph, 2018). To test this for TLE patients with ATL resection, we used the Synonym Judgement Task, which varied frequency and imageability in a $2 \times 2$ design. The results showed that left but not right TLE patients showed a word frequency and imageability effect (i.e, poorer performance on low frequent as well as low imageability items). This aligns with the results of Rice et al. (2018). In contrast, Lambon Ralph et al. (2012) found that both left and right TLE patients with lobectomy were impaired on this comprehension task. A possible 
influencing factor of the current study could be that we used control participants with few years of education to match the patients. Compared to the study of Lambon Ralph et al. (2012), this resulted in relatively poor performance of the control group, making it more likely to classify the right TLE patients as semantically unimpaired.

However, Lambon Ralph et al. (2012) showed that even on standard semantic tests, patients perform slower compared to controls and attributed this to an underlying semantic problem. It is therefore important to examine reaction time as well as accuracy data. The reaction time data of the current study found impairments for left but not right TLE patients on the Synonym Judgement Task. This seems to strengthen the conclusion that right TLE patients are not affected on this verbal comprehension task.

Overall, even though previous research indicates that verbal comprehension might be more bilaterally represented, the current results suggest a left lateralisation.

\subsection{Episodic memory}

Episodic verbal memory was impaired in left but not right TLE patients. Episodic memory problems and material-specific lateralization effects are well described in the literature (e.g., Sherman et al., 2011; Sidhu et al., 2013; Witt et al., 2015; Zhao et al., 2014). More interestingly, the current study found correlations between episodic memory and performance on the Cambridge Naming Test for left TLE patients. This is not surprising as previous research provides evidence for a strong association between certain episodic and semantic memories (Kazui, Hashimoto, Hirono, \& Mori, 2003; Ratcliff \& McKoon, 1986; Snowden, Griffiths, \& Neary, 1996; Tulving 1986). Moreover, a study of Small and Sandhu (2008) suggests that picture naming seems to comprise both semantic and episodic aspects. Furthermore, the current results showed that the correlation between delayed recall and the Cambridge Naming Test was restricted to left TLE patients, which suggest that these episodic and semantic processes depend on the left medial temporal lobe. However, further research is required to interpret this.

\subsection{Neural reorganisation of the semantic system}

The current results showed that age of diagnosis was negatively correlated with the Cambridge Naming Test and the Synonym Judgement Task in left TLE patients. This 
means that an earlier diagnosis coincides with a better performance on these tasks, and seems contrary to literature suggesting that an early age at seizure onset is linked to poorer cognitive function (for review see: Breuer et al., 2016). However, with respect to language functions the patterns seems to be reversed in left TLE patients: an early seizure onset is linked to better language function (for review see: Goldmann \& Golby, 2005). In more detail, this review proposes a preoperative language transfer to the right hemisphere, thereby preserving normal language function and, in addition, that this reorganisation is more extensive in patients with an early seizure onset (see Springer et al., 1999). In other words, the young brain is more adaptable and reorganisation after damage due to seizures is more successful. In addition, the Cambridge Naming Test was positively correlated with months post-surgery, suggesting that naming performance improves over time after surgery, which is in line with a previous study testing the same patients before and at several stages after surgery (Giovagnoli et al., 2016). Although their study showed an initial decline in naming performance, patients improved over time.

\subsection{Conclusions}

By using a semantic battery with a comprehension and expressive task on both low and high frequency concepts, we obtained a clear picture of the semantic impairments in operated TLE patients. First, although naming and word finding difficulties are present on high frequency items (both in the current study and the literature), when looking at the individual results, it is clear that tests on low frequency items are more likely to classify a patient as impaired. This is important for clinical purposes, where we are often more interested in individual and not group results. Second, receptive semantic tasks, such as the Synonym Judgement Task clearly require low frequency words to detect semantic impairments. In addition, lateralisation effects become apparent because of the low frequency items. Finally, the inclusion of two tasks that probe semantics is important as this assures that we are revealing semantic impairments: if decreased performance is caused by a semantic impairement we would expect impaired patients to perform badly on both tasks.

Overall the results showed that although semantic memory is relatively preserved, it is clear that it is affected to a certain extent, which becomes apparent when using low frequent items. Furthermore, decreased performance was only apparent in the left 
resected TLE group on both the expressive (i.e., Cambridge and Specific Naming Tests) and comprehension (i.e., Synonym Judgement Task). This indicates that for verbal information, the bilateral semantic system is more sensitive to damage in the left compared to the right ATL, which is in line with theories that attribute a more prominent role to the left ATL due to connections with pre-semantic verbal regions (Binney, Parker, \& Lambon Ralph, 2012; Rice, Caswell, Moore, Hoffman, \& Lambon Ralph, 2018; Rice, Hoffman, \& Lambon Ralph, 2015; Rice, Lambon Ralph, \& Hoffman, 2015; Visser \& Lambon Ralph, 2011).

\subsection{Limitations of the current study}

The main goal of the study was to examine semantic impairments after unilateral ATL damage in resected TLE patients. Like any real neurological disease, interpretation of patients' behaviour is a complex task. The patients' behavioural profile is potentially influenced by a range of other factors beyond simply the acute neurological event itself, including the fact that resected TLE patients have suffered from long-standing preoperative neural changes caused by the epileptic seizures themselves and also patients can adopt positive compensatory strategies (Wise, 2003). Therefore, important insight can be gained by compairing pre- and post operative results (similar to Giovagnoli et al., 2016). Such a longitudinal study can reveal the extent of damage to the semantic system caused by the operation which cannot be attributed to pre-operative neural changes.

Another limitation is the inclusion of two naming tasks with different picture properties. Whereas the Cambridge Naming Test has black and white drawings, the Specific Naming Test included coloured photographs from the internet. This includes a confounding factor of general task difficulty on top of the word frequency difference between the two naming tasks.

Finally, modest deficits in right TLE patients might be missed due to the small sample sizes of the current study (i.e., 17 and 15 left and right TLE patients, respectively), leading to a lack of power. For example, the right TLE group performed somewhere in-between the left TLE and control groups on most tasks. The samples are small and negative findings do not definitively show a lack of effect. We therefore suggest that although the semantic impairments are more clear for left TLE patients, 
right TLE patients might be affected to a certain extent, which aligns with the hub-andspoke's model advocating graded, instead of absolute, hemispheric specialisation.

\subsection{Future research}

The current study has not focused on the role of the right ATL in semantic memory. To study this in more detail, future research could use non-verbal comprehension (on low frequency concepts) as this is especially bilaterally represented without the left $>$ right dominance (Visser \& Lambon Ralph, 2011; Visser, Jefferies, Embleton, \& Lambon Ralph, 2012). Similarly, it has been proposed that the semantic system in the right hemisphere is involved in processing weak semantic representations as well as atypical concepts and concentrating on these aspects could reveal more about the role of the right ATL and outcomes after right ATL damage in TLE patients (Faust \& Lavidor, 2003; Harpaz, Levkovitz, \& Lavidor, 2009; Jung-Beeman, 2005; Passeri, Capotosto, \& Di Matteo, 2015).

\section{References}

Barr, W. B., Goldberg, E., Wasserstein, J., \& Novelly, R. A. (1990). Retrograde amnesia following unilateral temporal lobectomy. Neuropsychologia, 28(3), 243255. http://doi.org/10.1016/0028-3932(90)90018-J

Bell, B. D., \& Giovagnoli, A. R. (2007). Recent Innovative Studies of Memory in Temporal Lobe Epilepsy. Neuropsychology Review, 17(4), 455-476. http://doi.org/10.1007/s11065-007-9049-3

Bell, B. D., Hermann, B. P., Woodard, A. R., Jones, J. E., Rutecki, P. A., Sheth, R., ... Seidenberg, M. (2001). Object naming and semantic knowledge in temporal lobe epilepsy. Neuropsychology, 15(4), 434-443. http://doi.org/10.1037//08944105.15.4.434

Binney, R. J., \& Lambon Ralph, M. A. (2015). Using a combination of fMRI and anterior temporal lobe rTMS to measure intrinsic and induced activation changes across the semantic cognition network. Neuropsychologia, 76, 170-181. http://doi.org/https://doi.org/10.1016/j.neuropsychologia.2014.11.009

Binney, R. J., Parker, G. J. M., \& Lambon Ralph, M. A. (2012). Convergent connectivity and graded specialization in the rostral human temporal lobe as revealed by diffusion-weighted imaging probabilistic tractography. Journal of Cognitive Neuroscience, 24(10), 1998-2014. http://doi.org/10.1162/jocn_a_00263

Bozeat, S., Lambon Ralph, M. A., Patterson, K., Garrard, P., \& Hodges, J. R. (2000). Non-verbal semantic impairment in semantic dementia. Neuropsychologia, 38(9), 1207-1215. http://doi.org/10.1016/S0028-3932(00)00034-8

Breuer, L. E. M., Boon, P., Bergmans, J. W. M., Mess, W. H., Besseling, R. M. H., de Louw, A., ... Aldenkamp, A. P. (2016). Cognitive deterioration in adult epilepsy: Does accelerated cognitive ageing exist? Neuroscience \& Biobehavioral Reviews, 
64, 1-11. http://doi.org/10.1016/j.neubiorev.2016.02.004

Butler, C. R., \& Zeman, A. Z. (2008). Recent insights into the impairment of memory in epilepsy: transient epileptic amnesia, accelerated long-term forgetting and remote memory impairment. Brain : A Journal of Neurology, 131(Pt 9), 2243-63. http://doi.org/10.1093/brain/awn127

Capitani, E., Laiacona, M., Mahon, B., \& Caramazza, A. (2003). What are the facts of semantic category-specific deficits? A critical review of the clinical evidence. Cognitive Neuropsychology, 20(3-6), 213-261. http://doi.org/10.1080/02643290244000266

Damasio, A. R. (1989). Time-locked multiregional retroactivation: A systems-level proposal for the neural substrates of recall and recognition. Cognition, 33(1), 2562. http://doi.org/10.1016/0010-0277(89)90005-X

Davies, K. G., Bell, B. D., Bush, A. J., Hermann, B. P., Curtis Dohan, F., \& Jaap, A. S. (1998). Naming Decline After Left Anterior Temporal Lobectomy Correlates with Pathological Status of Resected Hippocampus. Epilepsia, 39(4), 407-419. http://doi.org/10.1111/j.1528-1157.1998.tb01393.x

Devlin, J. T., Russell, R. P., Davis, M. H., Price, C. J., Wilson, J., Moss, H. E., ... Tyler, L. K. (2000). Susceptibility-Induced Loss of Signal: Comparing PET and fMRI on a Semantic Task. NeuroImage, 11(6), 589-600. http://doi.org/10.1006/nimg.2000.0595

Drane, D. L., Ojemann, J. G., Phatak, V., Loring, D. W., Gross, R. E., Hebb, A. O., ... Tranel, D. (2013). Famous face identification in temporal lobe epilepsy: support for a multimodal integration model of semantic memory. Cortex; a Journal Devoted to the Study of the Nervous System and Behavior, 49(6), 1648-67. http://doi.org/10.1016/j.cortex.2012.08.009

Elliott, G., Isaac, C. L., \& Muhlert, N. (2014). Measuring forgetting: a critical review of accelerated long-term forgetting studies. Cortex, 54(100), 16-32. http://doi.org/10.1016/j.cortex.2014.02.001

Faust, M., \& Lavidor, M. (2003). Semantically convergent and semantically divergent priming in the cerebral hemispheres: lexical decision and semantic judgment. Cognitive Brain Research, 17(3), 585-597. http://doi.org/10.1016/S09266410(03)00172-1

Gabrieli, J. D. E., Cohen, N. J., \& Corkin, S. (1988). The impaired learning of semantic knowledge following bilateral medial temporal-lobe resection. Brain and Cognition, 7(2), 157-177. http://doi.org/https://doi.org/10.1016/02782626(88)90027-9

Garrard, P., Lambon Ralph, M. A., Hodges, J. R., \& Patterson, K. (2001). Prototypicality, distinctiveness, and intercorrelation: Analyses of the semantic attributes of living and nonliving concepts. Cognitive Neuropsychology, 18(2), 125-174. http://doi.org/10.1080/02643290125857

Ghasemi, A., \& Zahediasl, S. (2012). Normality Tests for Statistical Analysis: A Guide for Non-Statisticians. International Journal of Endocrinology and Metabolism, 10(2), 486-489. http://doi.org/10.5812/ijem.3505

Giovagnoli, A. R., Erbetta, A., Villani, F., \& Avanzini, G. (2005). Semantic memory in partial epilepsy: verbal and non-verbal deficits and neuroanatomical relationships. Neuropsychologia, 43(10), 1482-92. http://doi.org/10.1016/j.neuropsychologia.2004.12.010

Giovagnoli, A. R., Parente, A., Didato, G., Manfredi, V., Deleo, F., Tringali, G., \& Villani, F. (2016a). The course of language functions after temporal lobe epilepsy surgery: a prospective study. European Journal of Neurology, 23(12), 1713-1721. 
http://doi.org/10.1111/ene.13113

Giovagnoli, A. R., Parente, A., Didato, G., Manfredi, V., Deleo, F., Tringali, G., \& Villani, F. (2016b). The course of language functions after temporal lobe epilepsy surgery: a prospective study. European Journal of Neurology, 23(12), 1713-1721. http://doi.org/10.1111/ene.13113

Goldmann, R. E., \& Golby, A. J. (2005). Atypical language representation in epilepsy: Implications for injury-induced reorganization of brain function. Epilepsy \& Behavior, 6(4), 473-487. http://doi.org/10.1016/j.yebeh.2005.03.012

Hamberger, M. J. (2015). Object naming in epilepsy and epilepsy surgery. Epilepsy \& Behavior, 46, 27-33. http://doi.org/10.1016/j.yebeh.2014.12.019

Harpaz, Y., Levkovitz, Y., \& Lavidor, M. (2009). Lexical ambiguity resolution in Wernicke's area and its right homologue. Cortex, 45(9), 1097-1103. http://doi.org/10.1016/j.cortex.2009.01.002

Hermann, B. (2015). Object naming in epilepsy. Epilepsy \& Behavior, 46, 10-11. http://doi.org/https://doi.org/10.1016/j.yebeh.2015.01.028

Hickok, G., \& Poeppel, D. (2004). Dorsal and ventral streams: a framework for understanding aspects of the functional anatomy of language. Cognition, 92(1-2), 67-99. http://doi.org/10.1016/j.cognition.2003.10.011

Hoffman, P., Binney, R. J., \& Lambon Ralph, M. A. (2015). Differing contributions of inferior prefrontal and anterior temporal cortex to concrete and abstract conceptual knowledge. Cortex, 63, 250-66. http://doi.org/10.1016/j.cortex.2014.09.001

Humphreys, G. F., Hoffman, P., Visser, M., Binney, R. J., \& Lambon Ralph, M. A. (2015). Establishing task- and modality-dependent dissociations between the semantic and default mode networks. Proceedings of the National Academy of Sciences of the United States of America, 112(25), 7857-62. http://doi.org/10.1073/pnas.1422760112

Humphreys, G. W., \& Forde, E. M. (2001). Hierarchies, similarity, and interactivity in object recognition: "category-specific" neuropsychological deficits. The Behavioral and Brain Sciences, 24(3), 453-509. http://doi.org/11682799

Ison, M. J., Quian Quiroga, R., Fried, I., Akaike, H., Aminoff, E., Gronau, N., ... Suzuki, W. A. (2015). Rapid Encoding of New Memories by Individual Neurons in the Human Brain. Neuron, 87(1), 220-30.

http://doi.org/10.1016/j.neuron.2015.06.016

Ives-Deliperi, V. L., \& Butler, J. T. (2012). Naming outcomes of anterior temporal lobectomy in epilepsy patients: A systematic review of the literature. Epilepsy \& Behavior, 24(2), 194-198. http://doi.org/10.1016/j.yebeh.2012.04.115

Javidan, M. (2012). Electroencephalography in Mesial Temporal Lobe Epilepsy: A Review. Epilepsy Research and Treatment, 2012, 637430. http://doi.org/10.1155/2012/637430

Jefferies, E., Patterson, K., Jones, R. W., \& Lambon Ralph, M. A. (2009). Comprehension of concrete and abstract words in semantic dementia. Neuropsychology, 23(4), 492-499. http://doi.org/10.1037/a0015452

Jung-Beeman, M. (2005). Bilateral brain processes for comprehending natural language. Trends in Cognitive Sciences, 9(11), 512-518. http://doi.org/10.1016/j.tics.2005.09.009

Jung, J., \& Lambon Ralph, M. A. (2016). Mapping the Dynamic Network Interactions Underpinning Cognition: A cTBS-fMRI Study of the Flexible Adaptive Neural System for Semantics. Cerebral Cortex, 26(8), 3580-3590. http://doi.org/http://dx.doi.org/10.1093/cercor/bhw149

Kazui, H., Hashimoto, M., Hirono, N., \& Mori, E. (2003). Nature of personal semantic 
memory: evidence from Alzheimer's disease. Neuropsychologia, 41(8), 981-988. http://doi.org/https://doi.org/10.1016/S0028-3932(02)00322-6

Lah, S., Grayson, S., Lee, T., \& Miller, L. (2004). Memory for the past after temporal lobectomy: impact of epilepsy and cognitive variables. Neuropsychologia, 42(12), 1666-79. http://doi.org/10.1016/j.neuropsychologia.2004.04.008

Lah, S., \& Smith, M. Lou. (2015). Verbal memory and literacy outcomes one year after pediatric temporal lobectomy: A retrospective cohort study. Epilepsy \& Behavior, 44, 225-233. http://doi.org/https://doi.org/10.1016/j.yebeh.2014.12.040

Lambon Ralph, M. A. (2014). Neurocognitive insights on conceptual knowledge and its breakdown. Philosophical Transactions of the Royal Society of London. Series B, Biological Sciences, 369(1634), 20120392. http://doi.org/10.1098/rstb.2012.0392

Lambon Ralph, M. A., Ehsan, S., Baker, G. A., \& Rogers, T. T. (2012). Semantic memory is impaired in patients with unilateral anterior temporal lobe resection for temporal lobe epilepsy. Brain, 135(1), 242-58. http://doi.org/10.1093/brain/awr325

Lambon Ralph, M. A., Jefferies, E., Patterson, K., \& Rogers, T. T. (2017). The neural and computational bases of semantic cognition. Nat Rev Neurosci, 18(1), 42-55. http://doi.org/http://dx.doi.org/10.1038/nrn.2016.150

Lambon Ralph, M. A., McClelland, J. L., Patterson, K., Galton, C. J., \& Hodges, J. R. (2001). No right to speak? The relationship between object naming and semantic impairment: neuropsychological evidence and a computational model. Journal of Cognitive Neuroscience, 13(3), 341-56.

http://doi.org/http://dx.doi.org/10.1162/08989290151137395

Lambon Ralph, M. A., Pobric, G., \& Jefferies, E. (2009). Conceptual Knowledge Is Underpinned by the Temporal Pole Bilaterally: Convergent Evidence from rTMS. Cerebral Cortex, 19(4), 832-838. http://doi.org/10.1093/cercor/bhn131

Lou Smith, M., Elliott, I. M., \& Lach, L. (2006). Memory Outcome After Pediatric Epilepsy Surgery: Objective and Subjective Perspectives. Child Neuropsychology, 12(3), 151-164. http://doi.org/10.1080/09297040591001076

Martin, A. (2007). The Representation of Object Concepts in the Brain. Annual Review of Psychology, 58(1), 25-45.

http://doi.org/10.1146/annurev.psych.57.102904.190143

Messas, C. S., Mansur, L. L., \& Castro, L. H. M. (2008). Semantic memory impairment in temporal lobe epilepsy associated with hippocampal sclerosis. Epilepsy \& Behavior: E\&B, 12(2), 311-316. http://doi.org/10.1016/j.yebeh.2007.10.014

O'Shea, J., Sebastian, C., Boorman, E. D., Johansen-Berg, H., \& Rushworth, M. F. S. (2007). Functional specificity of human premotor-motor cortical interactions during action selection. European Journal of Neuroscience, 26(7), 2085-2095. http://doi.org/10.1111/j.1460-9568.2007.05795.x

Ojemann, L. M., Ojemann, G. A., Dodrill, C. B., Crawford, C. A., Holmes, M. D., \& Dudley, D. L. (2001). Language Disturbances as Side Effects of Topiramate and Zonisamide Therapy. Epilepsy \& Behavior, 2(6), 579-584. http://doi.org/10.1006/ebeh.2001.0285

Passeri, A., Capotosto, P., \& Di Matteo, R. (2015). The right hemisphere contribution to semantic categorization: A TMS study. Cortex, 64, 318-326. http://doi.org/10.1016/j.cortex.2014.11.014

Pobric, G., Jefferies, E., \& Lambon Ralph, M. A. (2010). Amodal semantic representations depend on both anterior temporal lobes: Evidence from repetitive transcranial magnetic stimulation. Neuropsychologia, 48(5), 1336-1342. http://doi.org/https://doi.org/10.1016/j.neuropsychologia.2009.12.036 
Pobric, G., Jefferies, E., \& Ralph, M. A. L. (2007). Anterior temporal lobes mediate semantic representation: mimicking semantic dementia by using rTMS in normal participants. Proceedings of the National Academy of Sciences of the United States of America, 104(50), 20137-41. http://doi.org/10.1073/pnas.0707383104

Ratcliff, R., \& McKoon, G. (1986). More on the distinction between episodic and semantic memories. Journal of Experimental Psychology: Learning, Memory, and Cognition. US: American Psychological Association. http://doi.org/10.1037/02787393.12.2.312

Rey, A. (1958). L'Examen Clinique en Psychologie (Press Univ). Paris.

Rice, G. E., Caswell, H., Moore, P., Hoffman, P., \& Lambon Ralph, M. A. (2018). The Roles of Left Versus Right Anterior Temporal Lobes in Semantic Memory: A Neuropsychological Comparison of Postsurgical Temporal Lobe Epilepsy Patients. Cerebral Cortex, 28(4), 1487-1501. http://doi.org/http://dx.doi.org/10.1093/cercor/bhx362

Rice, G. E., Hoffman, P., \& Lambon Ralph, M. A. (2015). Graded specialization within and between the anterior temporal lobes. Annals of the New York Academy of Sciences, 1359(1), 84-97. http://doi.org/10.1111/nyas.12951

Rice, G. E., Lambon Ralph, M. A., \& Hoffman, P. (2015). The Roles of Left Versus Right Anterior Temporal Lobes in Conceptual Knowledge: An ALE Meta-analysis of 97 Functional Neuroimaging Studies. Cerebral Cortex, 25(11), 4374-4391. http://doi.org/10.1093/cercor/bhv024

Rogers, T. T., Lambon Ralph, M. A., Garrard, P., Bozeat, S., McClelland, J. L., Hodges, J. R., \& Patterson, K. (2004). Structure and Deterioration of Semantic Memory: A Neuropsychological and Computational Investigation. Psychological Review, 111(1), 205-235. http://doi.org/10.1037/0033-295X.111.1.205

Schapiro, A. C., McClelland, J. L., Welbourne, S. R., Rogers, T. T., \& Lambon Ralph, M. A. (2013). Why Bilateral Damage Is Worse than Unilateral Damage to the Brain. Journal of Cognitive Neuroscience, 25(12), 2107-23. http://doi.org/10.1162/jocn_a_00441

Seidenberg, M., Griffith, R., Sabsevitz, D., Moran, M., Haltiner, A., Bell, B., ... Hermann, B. (2002). Recognition and identification of famous faces in patients with unilateral temporal lobe epilepsy. Neuropsychologia , 40(4), 446-456. http://doi.org/10.1016/S0028-3932(01)00096-3

Semah, F., Picot, M.-C., Adam, C., Broglin, D., Arzimanoglou, A., Bazin, B., ... Baulac, M. (1998). Is the underlying cause of epilepsy a major prognostic factor for recurrence? Neurology, 51(5), 1256-1262. http://doi.org/http://n.neurology.org/content/51/5/1256.abstract

Sherman, E. M. S., Wiebe, S., Fay-McClymont, T. B., Tellez-Zenteno, J., Metcalfe, A., Hernandez-Ronquillo, L., ... Jetté, N. (2011). Neuropsychological outcomes after epilepsy surgery: Systematic review and pooled estimates. Epilepsia, 52(5), 857869. http://doi.org/10.1111/j.1528-1167.2011.03022.x

Sidhu, M. K., Stretton, J., Winston, G. P., Bonelli, S., Centeno, M., Vollmar, C., ... Duncan, J. S. (2013). A functional magnetic resonance imaging study mapping the episodic memory encoding network in temporal lobe epilepsy. Brain, 136(6), 1868-1888. http://doi.org/10.1093/brain/awt099

Sierpowska, J., Gabarrós, A., Fernandez-Coello, A., Camins, À., Castañer, S., Juncadella, M., ... Rodríguez-Fornells, A. (2015). Morphological derivation overflow as a result of disruption of the left frontal aslant white matter tract. Brain and Language, 142, 54-64. http://doi.org/10.1016/j.bandl.2015.01.005

Simmons, W. K., \& Martin, A. (2009). The anterior temporal lobes and the functional 
architecture of semantic memory. Journal of the International Neuropsychological Society: JINS, 15(5), 645-9. http://doi.org/10.1017/S1355617709990348

Small, J. A., \& Sandhu, N. (2008). Episodic and semantic memory influences on picture naming in Alzheimer's disease. Brain and Language, 104(1), 1-9. http://doi.org/https://doi.org/10.1016/j.bandl.2006.12.002

Smith, M. Lou, \& Lah, S. (2011). One declarative memory system or two? The relationship between episodic and semantic memory in children with temporal lobe epilepsy. Neuropsychology, 25(5), 634-644. http://doi.org/10.1037/a0023770

Snowden, J. S., Griffiths, H. L., \& Neary, D. (1996). Semantic-Episodic Memory Interactions in Semantic Dementia: Implications for Retrograde Memory Function. Cognitive Neuropsychology, 13(8), 1101-1139.

http://doi.org/10.1080/026432996381674

Springer, J. A., Binder, J. R., Hammeke, T. A., Swanson, S. J., Frost, J. A., Bellgowan, P. S. F., ... Mueller, W. M. (1999). Language dominance in neurologically normal and epilepsy subjects: A functional MRI study. Brain, 122(11), 2033-2046. http://doi.org/10.1093/brain/122.11.2033

Vandenberghe, R., Price, C., Wise, R., Josephs, O., \& Frackowiak, R. S. J. (1996). Functional anatomy of a common semantic system for words and pictures. Nature, 383(6597), 254-256. http://doi.org/10.1038/383254a0

Visser, M., Embleton, K. V, Jefferies, E., Parker, G. J., \& Ralph, M. A. L. (2010). The inferior, anterior temporal lobes and semantic memory clarified: novel evidence from distortion-corrected fMRI. Neuropsychologia, 48(6), 1689-96. http://doi.org/10.1016/j.neuropsychologia.2010.02.016

Visser, M., Jefferies, E., Embleton, K. V, \& Lambon Ralph, M. A. (2012). Both the middle temporal gyrus and the ventral anterior temporal area are crucial for multimodal semantic processing: distortion-corrected fMRI evidence for a double gradient of information convergence in the temporal lobes. Journal of Cognitive Neuroscience, 24(8), 1766-78. http://doi.org/10.1162/jocn_a_00244

Visser, M., \& Lambon Ralph, M. A. (2011). Differential contributions of bilateral ventral anterior temporal lobe and left anterior superior temporal gyrus to semantic processes. Journal of Cognitive Neuroscience, 23(10), 3121-31. http://doi.org/10.1162/jocn_a_00007

Voltzenlogel, V., Despres, O., Vignal, J.-P., Steinhoff, B. J., Kehrli, P., \& Manning, L. (2006). Remote Memory in Temporal Lobe Epilepsy. Epilepsia, 47(8), 1329-1336. http://doi.org/10.1111/j.1528-1167.2006.00555.x

Voltzenlogel, V., Hirsch, E., Vignal, J.-P., Valton, L., \& Manning, L. (2015). Preserved anterograde and remote memory in drug-responsive temporal lobe epileptic patients. Epilepsy Research, 115, 126-132. http://doi.org/10.1016/j.eplepsyres.2015.06.006

Wise, R. J. S. (2003). Language systems in normal and aphasic human subjects: Functional imaging studies and inferences from animal studies. British Medical Bulletin, 65, 95-119. http://doi.org/10.1093/bmb/65.1.95

Witt, J.-A., Coras, R., Schramm, J., Becker, A. J., Elger, C. E., Blümcke, I., \& Helmstaedter, C. (2015). Relevance of hippocampal integrity for memory outcome after surgical treatment of mesial temporal lobe epilepsy. Journal of Neurology, 262(10), 2214-2224. http://doi.org/10.1007/s00415-015-7831-3

Zhao, F., Kang, H., You, L., Rastogi, P., Venkatesh, D., \& Chandra, M. (2014).

Neuropsychological deficits in temporal lobe epilepsy: A comprehensive review. Annals of Indian Academy of Neurology, 17(4), 374-82. http://doi.org/10.4103/0972-2327.144003 
